\title{
ON AN INVERSE PROBLEM FROM MAGNETIC RESONANCE ELASTIC IMAGING*
}

\author{
DAVID J. N. WALL ${ }^{\dagger}$, PETER OLSSON $^{\ddagger}$, AND ELIJAH E. W. VAN HOUTEN $\$$
}

\begin{abstract}
The imaging problem of elastography is an inverse problem. The nature of an inverse problem is that it is ill-conditioned. We consider properties of the mathematical map which describes how the elastic properties of the tissue being reconstructed vary with the field measured by magnetic resonance imaging (MRI). This map is a nonlinear mapping, and our interest is in proving certain conditioning and regularity results for this operator which occurs implicitly in this problem of imaging in elastography. In this treatment we consider the tissue to be linearly elastic, isotropic, and spatially heterogeneous. We determine the conditioning of this problem of function reconstruction, in particular for the stiffness function. We further examine the conditioning when determining both stiffness and density. We examine the Fréchet derivative of the nonlinear mapping, which enables us to describe the properties of how the field affects the individual maps to the stiffness and density functions. We illustrate how use of the implicit function theorem can considerably simplify the analysis of Fréchet differentiability and regularity properties of this underlying operator. We present new results which show that the stiffness map is mildly ill-posed, whereas the density map suffers from medium ill-conditioning. Computational work has been done previously to study the sensitivity of these maps, but our work here is analytical. The validity of the Newton-Kantorovich and optimization methods for the computational solution of this inverse problem is directly linked to the Fréchet differentiability of the appropriate nonlinear operator, which we justify.
\end{abstract}

Key words. magnetic resonance elastography, MRE, inverse problem, Fréchet differentiability, shear stiffness reconstruction, noninvasive palpation, tumor imaging

AMS subject classifications. 74J25, 45Q05, 45A05, 45E99

DOI. $10.1137 / 110832082$

1. Introduction. This paper is concerned with developing the mathematical theory specifying the inverse problem from magnetic resonance elastography (MRE). A unique feature of this medical imaging technique is that it measures the field internally.

Palpation has been used by medical practitioners over the centuries to detect regions in soft tissue of varying stiffness. Palpation is used as a diagnostic method because the mechanical properties of tissues are often dramatically affected by the presence of disease processes, such as cancer, inflammation, and fibrosis. Magnetic resonance (MR) elastography uses mechanical shear waves to assess the stiffness of soft tissue. MRE is currently in use for the detection of liver disease through measurement of liver stiffness $[15,4]$ and is under evaluation for early detection of breast tumors $[22,45,40]$ and for multiple uses in the brain $[14,13,18]$, including injury and brain disease $[20,51,50]$.

MRE is a relatively new technique in medicine first considered in [29], and it combines magnetic resonance imaging (MRI) with shear wave elastography so as to

\footnotetext{
* Received by the editors April 26, 2011; accepted for publication (in revised form) May 16, 2011; published electronically September 13, 2011.

http://www.siam.org/journals/siap/71-5/83208.html

${ }^{\dagger}$ Biomathematics Research Centre, Department of Mathematics \& Statistics, University of Canterbury, Christchurch 8140, New Zealand (David.Wall@canterbury.ac.nz).

${ }^{\ddagger}$ Department of Applied Mechanics, Chalmers University of Technology, Gothenburg SE-41296, Sweden (Peter.Olsson@chalmers.se).

$\S$ Department of Mechanical Engineering, University of Canterbury, Christchurch 8140, New Zealand (Eli.vanHouten@canterbury.ac.nz).
} 
measure the elastic field displacement within the soft tissue. A recent review of this emerging modality is [24]. MRE enables higher resolution imaging of soft tissue areas, when compared with MRI, for tissues that have low hydrogen atom count, and this typically occurs in the stiff tissues referred to above. The MRE technique can provide imaging of possible early cancer tissue, as the elastic moduli of tumors changes during their pathological evolution. The importance of early detection of cancer tumors is well known, and it is for this reason that much research is currently being performed on the MRE modality. Furthermore, MRE is being investigated for measurement of brain tissue stiffness information as it may be related to diseases such as Alzheimer's, hydrocephalus, brain cancer, and multiple sclerosis.

This paper addresses the issue of how much stiffness information can be reconstructed or, in other words, how ill-conditioned is the inverse problem of MRE stiffness reconstruction. So our primary concern is with the conditioning of the inverse problem. To determine this it is necessary to look at the mathematical mapping properties prescribed by the physical phenomenon, namely elastodynamics. The explicit representation of this nonlinear map for the problem considered here is not possible; rather the map is described implicitly by the equations of elastodynamics. To generate a representation of the linearization of this map we use the implicit function theorem, a technique we have used in the past [10].

The first problem to ask might be: How can we solve the inverse problem computationally? This is not addressed here, although some of our formulations could be utilized computationally. The standard techniques currently used for this inverse problem range from finite element-based optimization methods to direct operations made on filtered data $[39,23,33,46,47]$. Various further mathematical treatments of this inverse problem have also been made $[25,26,30,3,5,44,2]$.

In this paper we address the mathematical formulation of the inverse problem with the aim of producing theoretical results on the conditioning of the inverse problem to aid the understanding of the results obtained from the aforementioned computational techniques. Associated with this is the central question underlying our ability to compare different techniques for solving the imaging problem: How ill-conditioned is the inverse problem? All properties of the inverse problem are determined by the mathematical properties of the map from the measured displacement field to the material functions which are to be determined. This map is intrinsically tied to the associated map from material functions to the true displacement field. Mathematically the inverse map can be stated as $\mathbb{T}^{-1}: \mathbf{u} \rightarrow \nu$. Here $\mathbf{u}$ is the true vector displacement field, and $\nu$ is the set of functions that are to be reconstructed. It is important to realize that this map is in general nonlinear, and this is true for all the inverse problems considered here. Associated with the inverse map is the nonlinear map for the direct problem $\mathbb{T}: \nu \rightarrow \mathbf{u}$, which maps the material functions to the displacement field. This map cannot be written explicitly but is determined implicitly from the mathematical equations describing the elastodynamic problem. It should be observed that the associated problem of determination of $\mathbf{u}$ given $\nu$ is linear in the case considered here.

In many inverse problems the operator $\mathbb{T}$ is a smoothing operator, and by that we mean that $\nu$ can have have rapid variation or abrupt jumps, but the effect on the displacement field is minimal. Perhaps this can be stated more clearly using linearization and spatial frequency ideas by saying that the high frequency components of $\nu$ have minimal effect on $\mathbf{u}$, when compared to low frequency components. In the forward map this does not cause a problem in the calculation of $\mathbf{u}$, but when the map is inverted, as in the inverse problem, it is not so straightforward. Another way of putting this in function space terminology is that the operator $\mathbb{T}$ is a compact 


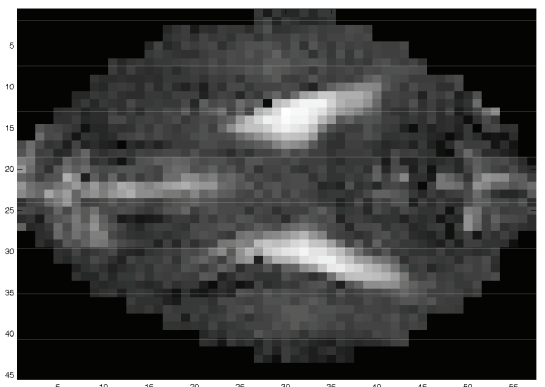

(a) T2 weighted MR image of the brain, showing the location of the fluid-filled ventricles.

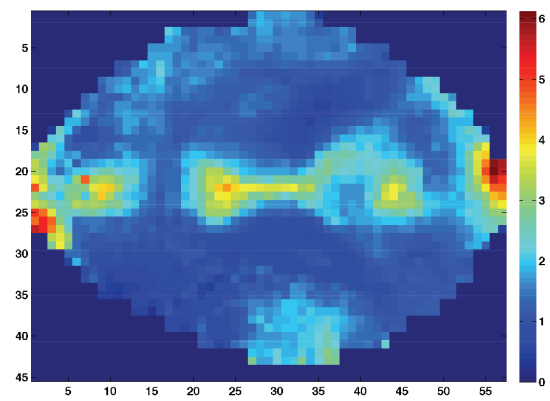

(c) Stiffness $\mu$ (real part) reconstruction, where the color bar shows units $\mathrm{kPa}$ for the shear modulus.

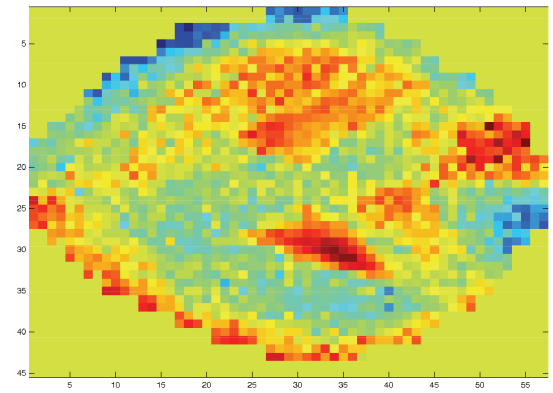

(b) $y$-component of the real part of the displacement, $\mathbf{u}$.

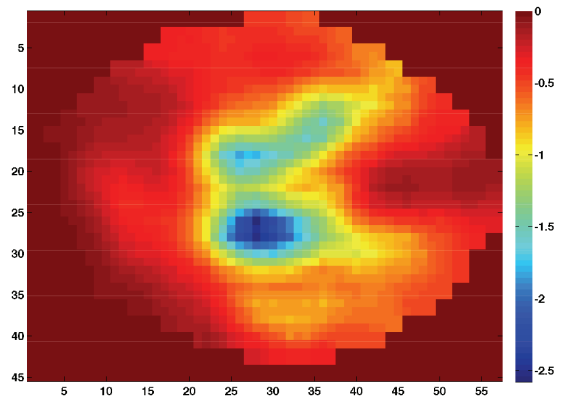

(d) Density (imaginary part), where the color bar shows units $10^{3} \mathrm{~kg} / \mathrm{m}^{2}$.

FIG. 1. Pictorial view of the mathematical map $\mathbb{T}^{-1}: \mathbf{u} \rightarrow \nu$, displayed from (b) to (c) for stiffness, and (b) to (d) for density. An MR image of the brain is shown in (a). Shown in (b) is the measured displacement field for a slice of the human brain, in (c) the reconstructed shear stiffness $\mu$, and in $(\mathrm{d})$ the reconstructed density $\rho$. The reason why the density is imaginary is because the brain soft tissue is almost incompressible; hence the Lamé parameter $\lambda$ is held constant at a real value $10^{8}$ while the reconstructed phase has an arbitrary baseline; so the loss component in $\lambda$ is shifted to the density. The reconstruction was made via finite element solution of the weak formulation of the equations of motion, and a regularized solution of a resulting optimization problem.

operator, and therefore for the inverse problem $\mathbb{T}^{-1}$ is unbounded. For the inverse problem of MRE this has big implications. We determine the conditioning of this problem of function reconstruction, particularly for the stiffness function. However, we also examine the conditioning when determining both stiffness and density. It should be pointed out that using the elastography technique for detection of cancer tumors in human tissue, stiffness relative contrast can be up to the order of $2000 \%$, whereas the density only varies on the order of $8 \%$ [36, 37, 42]. It is for this reason that stiffness is diagnostically more useful, and density is often not considered. In Figure 1 we illustrate this map. Figure 1(a) shows a T2 weighted MR image of the brain, showing the location of the fluid-filled ventricles, which are very low stiffness and have high damping. The reconstruction of the stiffness component $\mu$ is shown in Figure 1(c). The fluid-filled ventricles can be seen in the density reconstruction of 
Figure 1(d).

For solution of the inverse problem it is important to first appreciate that the map $\mathbb{T}$ should be at least continuous, and a further requirement which is necessary in order to utilize iterative computational techniques for this nonlinear problem is that the nonlinear map be differentiable. This paper addresses these properties of the map $\mathbb{T}^{-1}$.

In section 2 we derive the integral equation representation needed in the rest of the paper. The problem that we analyze here is effectively converted to a scattering problem so that we do not have to worry about body surface boundary effects. We claim that this makes no essential difference to the nature of our results, and it adds transparency in that it reduces the complexity of the calculations. The boundary effects are analyzed in another publication.

In section 3 analysis of the direct problem map is performed, and this enables us to derive the Fréchet derivatives of the nonlinear operator in section 4; these are given in (4.5b) and (4.14b), are central to our analysis, and also provide an explicit analytic expression for the partial Fréchet derivatives of the map $\mathbb{T}$. These are the underlying partial derivatives utilized in any Newton-Kantorovich method for solving the inverse problem. The full Fréchet differential is given in (4.19). Furthermore in section 4 the analysis of the conditioning of the inverse problem is found from these partial derivatives.

We shall define many integral operators in this paper, and the notation scheme we chose is to use the variations on the symbols $\mathbb{K}$ and $\mathbb{S}$ for weakly singular and singular integral operators, respectively.

2. Fundamental equations. Our concern here is with the problem when the media is isotropic, compressible, and linear. However, our interest is in applying this technique to more general media, so we keep the formulation as general as possible.

The mechanical state in the medium in $\mathbb{R}^{3}$, which has a spatially varying density $\rho(\mathbf{x})$ and elastic properties described by a spatially varying stiffness tensor $\mathbf{C}(\mathbf{x})$, is defined by the elastic state $\{\boldsymbol{\tau}, \mathbf{u}\}$, where $\mathbf{u}(\mathbf{x})$ denotes the elastic displacement of the media and $\boldsymbol{\tau}(\mathbf{x})$ denotes its resulting Cauchy stress tensor. The dynamic situation for time harmonic behavior, $\exp (i \omega t)$, with angular frequency $\omega$ in the medium is described by Navier's equation as

$$
\tau_{i j, i}+\omega^{2} \rho(\mathbf{x}) u_{j}=-\rho(\mathbf{x}) f_{j},
$$

where $\mathbf{f}$ is the body force producing the motion. Here we have used the comma notation to denote derivatives with respect to a Cartesian coordinate system; all subscripts in what follows are to take on values from $\{1,2,3\}$ as is appropriate for Cartesian coordinates in $\mathbb{R}^{3}$. We distinguish tensors and vectors from their components by utilizing the same symbol in a bold font. As the medium is elastic, the stress tensor is related to the displacement $\mathbf{u}$ by the stiffness tensor, $\mathbf{C}$, through Hooke's law as

$$
\tau_{i j}=C_{i j k \ell}(\mathbf{x}) u_{k, \ell}(\mathbf{x}) .
$$

Here we initially will assume that the medium is nothing more than a nonhomogeneous linearly elastic solid, thus ensuring that only the basic symmetry properties are satisfied by $\mathbf{C}$.

In order to write an integral representation for the displacement field in the medium we first introduce the Green state $\{\boldsymbol{\Sigma}, \mathbf{G}\}$, appropriate to a medium where the material parameters are homogeneous and where the Green displacement tensor 
$G_{i j}$ and its corresponding Green stress tensor $\Sigma_{i j k}$ satisfy

$$
\Sigma_{i j k, i}+\omega^{2} \rho^{0} G_{j k}=-\delta_{j k} \delta\left(\mathbf{x}-\mathbf{x}^{\prime}\right) .
$$

Once again the Green stress tensor is related to the Green displacement by the stiffness tensor through Hooke's law as

$$
\Sigma_{i j k}^{C^{0}}\left(\mathbf{x}, \mathbf{x}^{\prime}\right)=C_{i j \ell m}^{0} G_{\ell k, m}\left(\mathbf{x}, \mathbf{x}^{\prime}\right) .
$$

We emphasize here that the background material defining the state of the Green tensors, the mass density $\rho^{0}$, and stiffness tensor $\mathbf{C}^{0}$ is homogeneous with appropriate material constants. This Green stress tensor with a constant background material will be used extensively in this paper. It will be necessary to calculate the stress with a Green tensor through the formula (2.4) but with different stiffness tensors, and so, where it is necessary to assure the reader as to the dependence on a particular stiffness, we append a superscript on the stress symbol to denote the stiffness under discussion as in (2.4).

We should note that when taking derivatives of the Green functions, which are of two arguments, the derivative notation is as follows: if the derivative is with respect to a primed coordinate, then we denote it by an appropriate primed index.

In order to analyze various integral operators that appear in our analysis, knowledge of the properties of the kernels of the integral operators is essential. It turns out that these can be determined from knowledge of the Green function for an isotropic homogeneous medium, so for explicit representation we discuss here the Green state for an isotropic medium. Furthermore, as is made apparent in Appendix A.2, the dominant singularity of the kernels is determined by the static or time independent Green state. For completeness, the nature of this singularity is discussed further in Appendix A. The homogeneous isotropic medium can be described by the Lamé parameters $\lambda$ and $\mu$ so that the stiffness tensor becomes

$$
C_{i j k l}=\lambda \delta_{i j} \delta_{k \ell}+\mu\left(\delta_{i k} \delta_{j \ell}+\delta_{i \ell} \delta_{j k}\right) .
$$

Then by Fourier transformation of (2.3) and (2.4) a concrete representation for the Green tensors can be found as

$$
G_{i j}=\frac{1}{4 \pi \rho^{0} \omega^{2}}\left[\delta_{i k} k_{S}^{2} g^{k_{S}}\left(\mathbf{x} ; \mathbf{x}^{\prime}\right)-\partial_{i} \partial_{j}\left[g^{k_{P}}\left(\mathbf{x}, \mathbf{x}^{\prime}\right)-g^{k_{S}}\left(\mathbf{x}, \mathbf{x}^{\prime}\right)\right]\right],
$$

where $g^{k}\left(\mathbf{x}, \mathbf{x}^{\prime}\right)=e^{i k\left|\mathbf{x}-\mathbf{x}^{\prime}\right|} /\left|\mathbf{x}-\mathbf{x}^{\prime}\right|$. The pressure and shear wave numbers are given, respectively, by $k_{P}^{2}=\rho^{0} \omega^{2} /(\lambda+2 \mu)$ and $k_{S}^{2}=\rho^{0} \omega^{2} / \mu$.

We make the assumption that the heterogeneous region is a bounded simply connected open region $\Omega \subset \mathbb{R}^{3}$ such that the surface $S=\bar{\Omega} \backslash \Omega=\partial \Omega$ in $\mathbb{R}^{3}$. Then we will form our integral representation for the displacement field by the Betti-Rayleigh reciprocity principle which is derived via standard means by using the divergence theorem. Consider the tensor state formed from $\{\boldsymbol{\tau}, \mathbf{u}\}$ and $\{\boldsymbol{\Sigma}, \boldsymbol{G}\}$ as

$$
p_{i k}=\Sigma_{i j k} u_{j}-\tau_{i j} G_{j k},
$$

with the divergence of this quantity given by

$$
p_{i k, i}=\Sigma_{i j k, i} u_{j}+\Sigma_{i j k} u_{j, i}-\tau_{i j, i} G_{j k}-\tau_{i j} G_{j k, i} .
$$


Now using the symmetry properties satisfied by the stiffness tensor, it is seen that

$$
\tau_{i j} G_{j k, i}=C_{i j \ell m}(\mathbf{x}) G_{\ell k, m} u_{j, i} .
$$

With use of the Navier equations (2.1) and (2.3), with (2.4) plus (2.8) in (2.7), together with the divergence theorem applied over a region bounded by a sphere with surface $S_{\infty}$ of very large radius, and a sphere with surface $S_{\epsilon}$ of radius $\epsilon$, both centred about $\mathbf{x}^{\prime}$, we find that

$$
\begin{aligned}
\int_{S_{\epsilon} \cup S_{\infty}} t_{k}\left(\mathbf{x}, \mathbf{x}^{\prime}\right) d S= & \int_{\Omega} \rho(\mathbf{x}) f_{j}(\mathbf{x}) G_{j k}\left(\mathbf{x}, \mathbf{x}^{\prime}\right) d V+\omega^{2} \int_{\Omega}\left(\rho(\mathbf{x})-\rho^{0}\right) G_{j k}\left(\mathbf{x}, \mathbf{x}^{\prime}\right) u_{j}(\mathbf{x}) d V \\
& +\int_{\Omega}\left(C_{i j \ell m}^{0}-C_{i j \ell m}(\mathbf{x})\right) G_{\ell k, m}\left(\mathbf{x}, \mathbf{x}^{\prime}\right) u_{j, i}(\mathbf{x}) d V
\end{aligned}
$$

where the traction in the surface integral is $t_{k}\left(\mathbf{x}, \mathbf{x}^{\prime}\right)=\left[n_{i} \Sigma_{i j k}\left(\mathbf{x}, \mathbf{x}^{\prime}\right) u_{j}(\mathbf{x})-\right.$ $\left.n_{i} \tau_{i j}(\mathbf{x}) G_{j k}\left(\mathbf{x}, \mathbf{x}^{\prime}\right)\right]$ with the appropriate orientation of the normal vector $n_{i}$ on the surfaces. The first term on the right-hand side of this equation generates the incident displacement field in the medium $\Omega$, generated by the body sources $\mathbf{f}$, and we denote this field by $\mathbf{u}^{\text {inc }}$. It is assumed here that this field is known; however, this may not be the case without use of a dummy replacement. If this incident field is not known, it is possible to use the fact that on the boundary of $\Omega$ the surface tractions are zero. However, this further complicates the problem in that the fundamental Green state cannot be used, but not much of the analysis shown here is changed as the Green state is modified by only a nonsingular part. We further make the assumption that the field scattered by the enclosing surface $S$ is negligible; this assumption can be removed straightforwardly if $\Omega$ is enlarged to $\mathbb{R}^{3}$ and the fields are made to satisfy radiation conditions. With these observations we are enabled to write the integral representation $(2.9)$ as

$$
\begin{aligned}
u_{k}(\mathbf{x})= & u_{k}^{\mathrm{inc}}(\mathbf{x})+\omega^{2} \int_{\Omega} \bar{\rho}\left(\mathbf{x}^{\prime}\right)\left(\mathbf{x}^{\prime}\right) G_{j k}\left(\mathbf{x}^{\prime}, \mathbf{x}\right) u_{j}\left(\mathbf{x}^{\prime}\right) d V^{\prime} \\
& -\int_{\Omega} \bar{C}_{i j \ell m}\left(\mathbf{x}^{\prime}\right) G_{\ell k, m^{\prime}}\left(\mathbf{x}^{\prime}, \mathbf{x}\right) u_{j, i}\left(\mathbf{x}^{\prime}\right) d V^{\prime}
\end{aligned}
$$

where $\bar{\rho}=\rho-\rho^{0}$ is the difference density function and

$$
\bar{C}_{i j k \ell}(\mathbf{x})=C_{i j k \ell}(\mathbf{x})-C_{i j k \ell}^{0}
$$

is the difference stiffness tensor. The surface integral over $S_{\epsilon}$ about the point $\mathbf{x}^{\prime}=\mathbf{x}$ on the left-hand side of (2.9) provides the term on the left-hand side of (2.10); see Appendix A.4.

We observe that the kernels in the integrals in (2.10) have weak singularities, of order 1 and 2, as $\mathbf{x} \rightarrow \mathbf{x}^{\prime}$ in the first and second integrals, respectively, and so are integrable (see Appendix A.2). Equation (2.10) can be converted into an integrodifferential equation for $\mathbf{u}$ by restriction of $\mathbf{x} \in \bar{\Omega}$. It then provides a means of solving the direct problem of calculation of the displacement field within $\Omega$, given the material parameters and the incident field; this is considered extensively in section 3 . When the problem is two-dimensional and the stiffness tensor is isotropic, the equation can be solved as two uncoupled equations depending upon the nature of the incident field; for an isotropic two-dimensional Lamé material these equations have been solved numerically by [21] for spatially varying material functions. 
Later we will need to use the Green state $\{\widetilde{\boldsymbol{\Sigma}}, \widetilde{\mathbf{G}}\}$, appropriate to a medium where the material has the same heterogeneous properties as in (2.1) and (2.2) and where the Green displacement tensor $\widetilde{G}_{i j}$ and its corresponding Green stress tensor $\widetilde{\Sigma}_{i j n}$ satisfy

$$
\widetilde{\Sigma}_{i j n, i}+\omega^{2} \rho(\mathbf{x}) \widetilde{G}_{j n}=-\delta_{j n} \delta\left(\mathbf{x}-\mathbf{x}^{\prime \prime}\right) .
$$

Once again the Green stress tensor is related to the Green displacement by the stiffness tensor through Hooke's law as

$$
\widetilde{\Sigma}_{i j n}^{C}\left(\mathbf{x}, \mathbf{x}^{\prime \prime}\right)=C_{i j r s}(\mathbf{x}) \widetilde{G}_{r n, s}\left(\mathbf{x}, \mathbf{x}^{\prime \prime}\right) .
$$

We observe here that the differentiation operator is with respect to the first argument of $\widetilde{\Sigma}$, which is similar to (2.4). Then, by techniques similar to those used earlier, we can write the integral representation for the heterogeneous Green function in terms of the homogeneous Green function as

$$
\begin{aligned}
\widetilde{G}_{k n}\left(\mathbf{x}, \mathbf{x}^{\prime}\right)= & G_{n k}\left(\mathbf{x}^{\prime}, \mathbf{x}\right)+\omega^{2} \int_{\Omega} \bar{\rho}\left(\mathbf{x}^{\prime \prime}\right) G_{j k}\left(\mathbf{x}^{\prime \prime}, \mathbf{x}\right) \widetilde{G}_{j n}\left(\mathbf{x}^{\prime \prime}, \mathbf{x}^{\prime}\right) d V^{\prime \prime} \\
& -\int_{\Omega} \bar{C}_{i j r s}\left(\mathbf{x}^{\prime \prime}\right) G_{r k, s^{\prime \prime}}\left(\mathbf{x}^{\prime \prime}, \mathbf{x}\right) \widetilde{G}_{j n, i^{\prime \prime}}\left(\mathbf{x}^{\prime \prime}, \mathbf{x}^{\prime}\right) d V^{\prime \prime}, \quad \mathbf{x} \neq \mathbf{x}^{\prime},
\end{aligned}
$$

where the indicated partial derivatives are with respect to the first argument. Throughout the following we use the notation that primes on partial derivatives indicate differentiation with respect to the corresponding $\mathbf{x}^{\prime}$-variable. We observe that the two Green tensors in the first integral in this equation have weak singularities of order 1 , and so when $\mathbf{x}=\mathbf{x}^{\prime}$ this integral is integrable. The second integral is not defined when $\mathbf{x}=\mathbf{x}^{\prime}$; however, its integral with respect to either $\mathbf{x}$ or $\mathbf{x}^{\prime}$ over $\Omega$ is, and that is the context in which we use it in section 4 to obtain simple representations for the Fréchet derivative of the inverse problem maps.

We now briefly examine the symmetry properties satisfied by the Green tensors that will be useful in the rest of the paper. First we note that all the displacement and stress Green tensors previously defined are symmetric in both their indices and arguments; i.e., they satisfy equations of the form $\widetilde{\mathbf{G}}\left(\mathbf{x}, \mathbf{x}^{\prime}\right)=\widetilde{\mathbf{G}}\left(\mathbf{x}^{\prime}, \mathbf{x}\right), \widetilde{\mathbf{G}}_{i j}=\widetilde{\mathbf{G}}_{j i}$. Moreover, as the homogeneous Green tensor's defining equation satisfies the translational invariance property plus the symmetry condition on their argument, it follows that the argument of these tensors is $\left|\mathbf{x}-\mathbf{x}^{\prime}\right|$, and this will mean that they are not differentiable when $\mathbf{x}=\mathbf{x}^{\prime}$.

When using symmetry properties to examine (2.14), we observe that the $k$ and $n$ indices can be interchanged on the Green tensors in (2.14). Also, due to symmetry properties satisfied by the stiffness tensor, the index pairs $r s$ and $j i$ can be interchanged on the Green tensors in the second integral on the right-hand side of (2.14).

It is observed that, although we have not used any symmetry properties satisfied by the Green tensors in (2.10) and (2.14), they will be used extensively in the rest of the paper.

We next introduce various integral operators that are necessary in discussing the inverse problem and provide existence and uniqueness results for the various direct problems needed in section 4 .

3. Mathematical details of the direct problem. Prior to examination of the inverse problem it is necessary to set up appropriate notation and to assemble 
results concerning the various direct problems that are essential in our subsequent development.

The analysis in this paper is simplified by working in Sobolev spaces. Although many of our results could be extended to classical spaces, we do not attempt that here. Here our discussions are to present the difficulties and nature of MRE as a tool for probing structures in biological tissues. Much of this information is readily available through utilizing Sobolev space theory and pseudodifferential operator theory.

Prior to providing existence theory for the direct problem we must ensure that the direct problem as stated has a unique solution.

LEMma 3.1. The direct problem as stated in (2.1) and (2.2), for an isotropic stiffness tensor, has a unique solution with $\bar{C} \in C^{2}(\Omega)$.

Proof. This has been provided by [16] for the scattering problem as considered here; see also [48].

3.1. Difference stiffness tensor. We first consider the case when the difference stiffness is nonzero but the difference density is zero. Then from (2.10) an integrodifferential equation for the determination of $\mathbf{u}$ is

$$
\begin{aligned}
u_{k}(\mathbf{x}) & =u_{k}^{\mathrm{inc}}(\mathbf{x})-\int_{\Omega} \bar{C}_{i j \ell m}\left(\mathbf{x}^{\prime}\right) G_{\ell k, m^{\prime}}\left(\mathbf{x}^{\prime}, \mathbf{x}\right) u_{j, i}\left(\mathbf{x}^{\prime}\right) d V^{\prime} \\
& =u_{k}^{\mathrm{inc}}(\mathbf{x})-\left.\mathbb{K}_{\bar{C}} \mathbf{u}\right|_{k}, \quad \mathbf{x} \in \Omega .
\end{aligned}
$$

Notice that the weakly singular integral operator $\mathbb{K}$ defined in (3.1a) can be considered to map either $\bar{C}$ or $\mathbf{u}$, and we separate the two maps by appending the material property as a subscript to the integral operator; this will be important in later sections. The integral operator is therefore defined by

$$
\left.\mathbb{K}_{\bar{C}} \mathbf{u}\right|_{k}=\int_{\Omega} \Sigma_{i j k}^{\bar{C}}\left(\mathbf{x}^{\prime}, \mathbf{x}\right) u_{j, i}\left(\mathbf{x}^{\prime}\right) d V^{\prime}
$$

where we have used the difference stress tensor $\boldsymbol{\Sigma}^{\bar{C}}$, the stress tensor as defined in (2.4) but with the difference stiffness as defined in (2.11).

Notice that the kernel in (3.2) is weakly singular; in fact the singularity is of order 2 (see Appendix A). However, existence theory for the solution of (3.2) is not easily handled, so we shall look at properties of a regularized equation. We shall utilize this equation later in section 4 to analyze the derivative of the map $\mathbb{T}$.

Equation (3.1a) can be converted into an integral equation for the Jacobian $w_{k r}=$ $u_{k, r}$ by differentiation of both sides of the equation; this is a left regularizer. Rather than work with the resulting $3^{2}$ equations, we choose to work with a right regularizer for (3.1a), and this corresponds to integration of the integral term by parts, so we arrive at

$$
\begin{aligned}
u_{k}(\mathbf{x}) & +\int_{S_{\epsilon} \cup S_{\infty}} n_{i} \Sigma_{i j k}^{\bar{C}}\left(\mathbf{x}, \mathbf{x}^{\prime}\right) u_{j} d S^{\prime} \\
= & u_{k}^{\mathrm{inc}}(\mathbf{x})+\int_{\Omega} \partial_{i^{\prime}}\left[\bar{C}_{i j \ell m}\left(\mathbf{x}^{\prime}\right) G_{\ell k, m^{\prime}}\left(\mathbf{x}^{\prime}, \mathbf{x}\right)\right] u_{j}\left(\mathbf{x}^{\prime}\right) d V^{\prime} \\
= & u_{k}^{\mathrm{inc}}(\mathbf{x})+\int_{\Omega} \Sigma_{i j k, i^{\prime}}^{\bar{C}}\left(\mathbf{x}^{\prime}, \mathbf{x}\right) u_{j}\left(\mathbf{x}^{\prime}\right) d V^{\prime}, \quad \mathbf{x} \in \Omega .
\end{aligned}
$$

Here the kernel of the volume integral has a singularity of order 3 (see Appendix A), and so the integral on the right-hand side must be treated as a singular operator. 
Now, on performing the surface integrations in (3.3) (see Appendix A.4), we find

$$
\alpha u_{k}(\mathbf{x})-\int_{\Omega} \Sigma_{i j k, i^{\prime}}^{\bar{C}}\left(\mathbf{x}^{\prime}, \mathbf{x}\right) u_{j}\left(\mathbf{x}^{\prime}\right) d V^{\prime}=u_{k}^{\mathrm{inc}}(\mathbf{x}), \quad \mathbf{x} \in \Omega,
$$

where $\alpha(\overline{\mathbf{C}}(\mathbf{x}))>0$ is a function of the difference stiffness tensor $\overline{\mathbf{C}}(\mathbf{x})$ and it is evaluated in the next section for an isotropic medium. The integral in (3.4) defines the integral operator

$$
\left.\mathbb{S}_{\bar{C}}(\cdot)\right|_{k}=\int_{\Omega} \Sigma_{i j k, i^{\prime}}^{\bar{C}}\left(\mathbf{x}^{\prime}, \mathbf{x}\right)(\cdot)_{j} d V^{\prime},
$$

which we will examine in detail in the next section.

We have presented in this section three different formulations for solution of the direct problem. We choose to develop existence theory for the direct problem specified from the singular integral representation given by (3.4). Then the solution $\mathbf{u}$ to the direct problem of determination of the scattered displacement field from knowledge of the incident field and the difference stiffness can be found from the solution of the second kind singular integral

$$
\left(\alpha \mathbb{I}-\mathbb{S}_{\bar{C}}\right) \mathbf{u}=\mathbf{u}^{\mathrm{inc}}, \quad \mathbf{x} \in \Omega .
$$

It is important to observe at this stage that it is not the direct problem with which we are mainly concerned here but rather the inverse problem, but we need appropriate equations on which to be able to apply the implicit function theorem, thus yielding results for the inverse problem.

3.1.1. Isotropic stiffness tensor and integral equation solution. We restrict the formulation in this section to the case when the stiffness tensor is of the form

$$
C_{i j \ell m}=\lambda(\mathbf{x}) \delta_{i j} \delta_{\ell m}+\mu(\mathbf{x})\left(\delta_{i \ell} \delta_{j m}+\delta_{i m} \delta_{j \ell}\right),
$$

which shows explicitly that there are two spatially varying functions describing the stiffness in the medium, which we denote generically by the set $\sigma=\{\lambda, \mu\}$. In what follows we need the differences of the Lamé functions $\bar{\lambda}(\mathbf{x})=\lambda(\mathbf{x})-\lambda^{0}$ and $\bar{\mu}(\mathbf{x})=\mu(\mathbf{x})-\mu^{0}$, but throughout this section $\bar{\sigma}$ takes on only one of its possible values; the other one is assumed to be zero. The case for both differing from zero is treated in section 3.3.

Now we define, from the vector-valued integral operator appearing in (3.6), the operators

$$
\left.\mathbb{S}_{\bar{\sigma}} \mathbf{u}\right|_{k}=\int_{\Omega} \Sigma_{i j k, i^{\prime}}^{\bar{\sigma}}\left(\mathbf{x}^{\prime}, \mathbf{x}\right) u_{j}\left(\mathbf{x}^{\prime}\right) d V^{\prime}, \quad \bar{\sigma}=\{\bar{\lambda}, \bar{\mu}\} .
$$

We have appended the subscript $\bar{\sigma}$ to the integral operator $\mathbb{S}_{\bar{\sigma}}$ because this integral operator can be considered to have two operands, first the material function $\bar{\sigma}$ and second the displacement field. Observe that we have in effect defined two integral operators here through $\bar{\sigma}$. The specific form of the divergence of the difference stress tensor, defined through (2.4) but with stiffness (2.11) and appearing in (3.8), is

$$
\Sigma_{i j k, i^{\prime}}^{\bar{\sigma}}=S_{k j}^{\lambda}(\bar{\lambda})+S_{k j}^{\mu}(\bar{\mu})
$$


where the $S$, the kernels of the integral operators $\mathbb{S}_{\bar{\sigma}}$, are also considered as linear operators on $\bar{\sigma}$ and are given by

$$
\begin{aligned}
S_{k j}^{\lambda}(\cdot) & =\left(G_{\ell k, \ell^{\prime}} \partial_{j}+G_{\ell k, \ell^{\prime} j^{\prime}}\right)(\cdot)\left(\mathbf{x}^{\prime}, \mathbf{x}\right), \\
S_{k j}^{\mu}(\cdot) & =\left(\left(G_{i k, j^{\prime}}+G_{j k, i^{\prime}}\right) \partial_{i^{\prime}}+\left(G_{i k, j^{\prime} i^{\prime}}+G_{j k, i^{\prime} i^{\prime}}\right)\right)(\cdot)\left(\mathbf{x}^{\prime}, \mathbf{x}\right) .
\end{aligned}
$$

Observe that in these kernels a partial derivative operator operates on $\bar{\sigma}$ because we took the derivative of $\mathbf{u}$ in (3.1a), and so for the case considered here (3.4) can be rewritten by utilizing the definition of (3.8) as

$$
\left(\alpha \mathbb{I}-\mathbb{S}_{\bar{\sigma}}\right) \mathbf{u}=\mathbf{u}^{\mathrm{inc}}, \quad \bar{\sigma}=\{\bar{\lambda}, \bar{\mu}\}, \quad \mathbf{x} \in \Omega .
$$

The function $\alpha$ is given in (A.9) in Appendix A.4, and from this we see that with positive difference material functions $\alpha>1$. Furthermore, examination of (A.9) shows that the requirement that $\alpha>0$ amounts to the requirement that the wave equation for inhomogeneous material be elliptic, an obvious requirement. We observe that when both of these difference functions become zero, (3.11) becomes trivial.

We require that $\bar{\sigma} \in X^{\bar{\sigma}}$, with $X^{\bar{\sigma}}=\left\{\bar{\sigma}: \bar{\sigma} \in \mathrm{H}^{1}(\bar{\Omega}), 0 \leq \bar{\sigma}<M\right\}$. We now quote the existence and mapping properties for the right regularized problem (3.11).

THEOREM 3.2. For the direct problem with the only nonzero material difference function the isotropic stiffness tensor, with $\bar{\sigma}=\{\bar{\lambda}, \bar{\mu}\}$, and if $\bar{\sigma} \in X^{\bar{\sigma}}$, $\mathbf{u}^{\text {inc }} \in H^{0}(\bar{\Omega})^{3}$, then there exists a unique solution $\mathbf{u} \in H^{0}(\bar{\Omega})^{3}$ of (3.11). Moreover,

$$
\|\mathbf{u}\|_{H^{0}(\bar{\Omega})^{3}} \leq C\left\|\mathbf{u}^{i n c}\right\|_{H^{0}(\bar{\Omega})^{3}}
$$

for some constant $C$.

See Appendix B.1 for the proof. We observe that the stiffness functions in this theorem are in the weak derivative space $X^{\bar{\sigma}}$, and this will imply weak continuity of displacement and traction throughout $\Omega$. If the stiffness properties of the tissue being modeled do not allow for this, then surface integrals accounting for this will appear in our equations, such as (2.9), and the integral equation (3.11) will be modified.

The weakly singular formulation given by (3.1a) is important in section 4 . So equivalently, for this isotropic medium, we can define from (3.1a) the vector-valued operator

$$
\left.\mathbb{K}_{\bar{\sigma}} \mathbf{u}\right|_{k}=\int_{\Omega} \Sigma_{i j k}^{\bar{\sigma}}\left(\mathbf{x}^{\prime}, \mathbf{x}\right) u_{j, i}\left(\mathbf{x}^{\prime}\right) d V^{\prime}, \quad \bar{\sigma}=\{\lambda, \mu\},
$$

from (3.2). It should be observed that $\mathbb{K}_{\bar{\sigma}}$ is a linear integral operator on $\bar{\sigma}$ and a integro-differential operator on $\mathbf{u}$. The specific form of the stress tensor defined in (2.4), but with isotropic stiffness given by (3.7), is

$$
\Sigma_{i j k}^{\bar{\sigma}}=\bar{\lambda} k_{i j k}^{\lambda}+\bar{\mu} k_{i j k}^{\mu}
$$

where the kernels $k$ of the operator $\mathbb{K}_{\bar{\sigma}}$ are now

$$
k_{i j k}^{\lambda}=G_{\ell k, \ell^{\prime}} \delta_{i j}, \quad k_{i j k}^{\mu}=\left(G_{i k, j^{\prime}}+G_{j k, i^{\prime}}\right) .
$$

We note for future use that when the above sets of kernels are slightly modified by replacement of $\mathbf{G}$ by $\widetilde{\mathbf{G}}$ in their representation, and the order of the argument of $\widetilde{\mathbf{G}}$ is interchanged, we surmount the symbol with a tilde, such as $\widetilde{k}$.

We observe that, similarly to our previous notation, the subscript $\bar{\sigma}$ is attached to the integral operator $\mathbb{K}_{\bar{\sigma}}$ as an operand because this integral operator can be 
considered to have two operands. So for the case considered here, (3.1a) can be written

$$
\left(\mathbb{I}+\mathbb{K}_{\bar{\sigma}}\right) \mathbf{u}=\mathbf{u}^{\mathrm{inc}}, \quad \bar{\sigma}=\{\bar{\lambda}, \bar{\mu}\}, \quad \mathbf{x} \in \Omega .
$$

We make the comment that although we derived the integral equation (3.11) after (3.1b), it makes more sense to think of (3.11) as the fundamental equation and (3.15) as the right regularized equation.

To study the inverse problem we first need existence and regularity results for the direct problem when one of the stiffness differences differs from zero in turn, and this is provided in the next result. First we can reduce the requirements on $\bar{\sigma}$, as in this formulation there is no differential operator on $\bar{\sigma}$, and now require $X^{\bar{\sigma}}=\{\bar{\sigma}: \bar{\sigma} \in$ $\left.\mathrm{H}^{0}(\bar{\Omega}), 0 \leq \bar{\sigma}<M\right\}$.

COROLlaRY 3.3. For the direct problem with the nonzero material difference function being the isotropic stiffness tensor, and with $\bar{\sigma} \in X^{\bar{\sigma}}$ and $\mathbf{u}^{i n c} \in H^{0}(\bar{\Omega})^{3}$, there exists a unique solution $\mathbf{u} \in H^{1}(\bar{\Omega})^{3}$ of (3.15). Moreover,

$$
\|\mathbf{u}\|_{H^{1}(\bar{\Omega})^{3}} \leq C\left\|\mathbf{u}^{i n c}\right\|_{H^{0}(\bar{\Omega})^{3}}
$$

for some constant $C$.

Proof. We observe for the right regularization of (3.1a), as given by (3.11), that every solution of (3.11) is a solution of (3.1a), but to avoid missing some solutions of (3.1a) we require the right regularizer to have an image which is dense in the domain of the original operator in (3.15); that this is so follows directly as the simplest function space that a solution of $(3.1 \mathrm{a})$ can be is $\mathrm{H}^{1}(\bar{\Omega})^{3}$. The bound on the inverse operator then follows directly from Theorem 3.2.

For use later in section 4.1.1 we notice that the operator defined in $\mathbb{K}_{\bar{\sigma}} \mathbf{u}$ for fixed $\mathbf{u}$ can be considered as a mapping of $\bar{\sigma}$. This follows by an argument similar to that in the proof of Theorem 3.4; it can be shown that it is a compact map of $\bar{\sigma}$ as $\mathbb{K} \mathbf{u}: X^{\bar{\sigma}} \rightarrow \mathrm{H}^{0}(\bar{\Omega})^{3}$.

3.2. Difference density. Often the reconstruction of the density is not done in MRE, but for completeness we consider it in this paper. When the difference density $\bar{\rho}$ differs from zero but the stiffness tensor is as the background stiffness $\mathbf{C}^{0}$, then from (2.10) an integral equation for the determination of $\mathbf{u}$ is

$$
u_{k}(\mathbf{x})=u_{k}^{\mathrm{inc}}(\mathbf{x})+\omega^{2} \int_{\Omega} \bar{\rho}\left(\mathbf{x}^{\prime}\right) G_{j k}\left(\mathbf{x}^{\prime}, \mathbf{x}\right) u_{j}\left(\mathbf{x}^{\prime}\right) d V^{\prime}, \quad \mathbf{x} \in \Omega .
$$

We define, from the previous equation, the vector-valued linear integral operators

$$
\left.\mathbb{R}(\cdot)\right|_{k}=\omega^{2} \int_{\Omega} G_{j k}\left(\mathbf{x}, \mathbf{x}^{\prime}\right)(\cdot)_{j} d V^{\prime}, \quad \mathbb{R}_{\bar{\rho}}(\cdot)=\mathbb{R}(\bar{\rho}(\mathbf{x}) \cdot),
$$

where we have used a restriction bar to denote the Cartesian component of the vectorvalued operator and also the symmetry property satisfied by G. Observe that the integral operator defined in (3.17) can be considered to have two operands, and in order to distinguish between these two, which we will need to in what follows, we append the material function as a subscript to the operator symbol in (3.18). Then the solution to the direct problem of determination of the scattered displacement field from knowledge of the incident field and the density difference is found from the solution of the linear Fredholm integral equation of the second kind,

$$
\left(\mathbb{I}-\mathbb{R}_{\bar{\rho}}\right) \mathbf{u}=\mathbf{u}^{\text {inc }}, \quad \mathbf{x} \in \Omega .
$$

Copyright (c) by SIAM. Unauthorized reproduction of this article is prohibited. 
3.2.1. Difference density and the integral equation solution. To study the inverse problem we first need existence and regularity results for the direct problem. These are provided by the results in this section.

THEOREM 3.4. For the direct problem with the only nonzero material difference function the density function and if $\rho \in X^{\rho}, X^{\rho}=\left\{\rho: \rho \in H^{0}(\bar{\Omega}), 0<\rho<M\right\}$, $\mathbf{u}^{\text {inc }} \in H^{0}(\bar{\Omega})^{3}$, then there exists a unique solution $\mathbf{u} \in H^{0}(\bar{\Omega})^{3}$ of (3.19). Moreover,

$$
\|\mathbf{u}\|_{H^{0}(\bar{\Omega})^{3}} \leq C\left\|\mathbf{u}^{i n c}\right\|_{H^{0}(\bar{\Omega})^{3}}
$$

for some constant $C$.

See Appendix B.2 for the proof.

We consider the operator $\mathbb{R}$ for fixed $\mathbf{u}$ as a mapping of $\bar{\rho}$, and furthermore define the operator $B_{\mathbf{u}}=\bar{\rho} \mathbf{u}$ for fixed $\mathbf{u}$ with $B_{\mathbf{u}}: X^{\rho} \rightarrow \mathrm{H}^{0}(\bar{\Omega})^{3}$ and note that $\mathbf{u}$ is bounded from the last theorem; it then follows that $\mathbb{R} \mathbf{u}$ is a compact map of $\bar{\rho}$ as $\mathbb{R} \mathbf{u}: X^{\rho} \rightarrow \mathrm{H}^{0}(\bar{\Omega})^{3}$. This fact will also be of use in section 4.2.1.

3.3. Nonzero difference stiffness and density for an isotropic medium. We now consider the combined direct problem of determination of $\mathbf{u}$ when the density and all stiffness functions, namely $\bar{\rho}(\mathbf{x})$ and $\bar{\sigma}(\mathbf{x})$, are known and differ from the Green state parameters. The integral equation for the determination of the displacement field in this case can be written from the formulations presented in the last two sections as

$$
\left(\alpha \mathbb{I}-\mathbb{R}_{\bar{\rho}}-\mathbb{S}_{\bar{\lambda}}-\mathbb{S}_{\bar{\mu}}\right) \mathbf{u}=\mathbf{u}^{\text {inc }}, \quad \mathbf{x} \in \Omega .
$$

Note that we have used the singular integral formulation for the stiffness functions. The analysis of this equation is similar to the case proven in Theorem 3.2. The existence and uniqueness of this problem is central to the elastography direct problem.

THEOREM 3.5. For the direct problem with nonzero material difference functions in density and isotropic stiffness, if $\bar{\rho} \in X^{\bar{\rho}}, \bar{\sigma} \in X^{\bar{\sigma}}$ in Theorem 3.2, and $\mathbf{u}^{\text {inc }} \in$ $H^{0}(\bar{\Omega})^{3}$, then there exists a unique solution $\mathbf{u} \in H^{0}(\bar{\Omega})^{3}$ of (3.21). Moreover,

$$
\|\mathbf{u}\|_{H^{0}(\bar{\Omega})^{3}} \leq C\left\|\mathbf{u}^{i n c}\right\|_{H^{0}(\bar{\Omega})^{3}}
$$

for some constant $C$.

See Appendix B.3 for the proof.

We will need the result that the weakly singular version of (3.21), as formulated in (2.10) and which we write as

$$
\left[\mathbb{I}-\mathbb{R}_{\bar{\rho}}+\mathbb{K}_{\lambda}+\mathbb{K}_{\mu}\right] \mathbf{u}(\mathbf{x})=\mathbf{u}^{\mathrm{inc}}(\mathbf{x}), \quad \mathbf{x} \in \Omega,
$$

has a unique bounded solution and this is provided by the following claim.

Corollary 3.6. For the direct problem with nonzero material difference functions in density and isotropic stiffness, if $\bar{\rho} \in X^{\bar{\rho}}, \bar{\sigma} \in X^{\bar{\sigma}}$ in Corollary 3.3, and $\mathbf{u}^{i n c} \in H^{0}(\bar{\Omega})^{3}$, then there exists a unique solution $\mathbf{u} \in H^{1}(\bar{\Omega})^{3}$ of (3.23). Moreover,

$$
\|\mathbf{u}\|_{H^{1}(\bar{\Omega})^{3}} \leq C\left\|\mathbf{u}^{i n c}\right\|_{H^{0}(\bar{\Omega})^{3}}
$$

for some constant $C$.

Proof. This follows from reasoning similar to that used for Corollary 3.3.

4. Inverse problems. The inverse problem of elastic constitutive function reconstruction is considered here. We will show that linearization of the various inverse operators is equivalent to an appropriate order of differentiation. Furthermore, it 
is shown that these inverse problems are always ill-posed for realistic measurement data. This is because the inverse maps are not onto, and the data that is measured can generally be placed only in the function space $L^{2}$, or at most $C$, and in these function spaces various differentiation operators are unbounded.

It is important to know whether the inverse problem is uniquely determined, and the answer to this is not straightforward because of the nonlinearity of the map $\mathbb{T}$. There have been a number of relevant results which show uniqueness for the inverse problem of determination of the elastic tensor, under certain conditions which we now discuss. The uniqueness of a 2-D inverse problem associated with elastic modulus imaging, given linearly independent displacement fields in an incompressible elastic material to determine the shear modulus, is discussed in [7]. Of more relevance here is the proof of uniqueness for the boundary measurement inverse problem to determine the isotropic shear modulus for three dimensions as given in [32]. In that paper it is required that one have boundary knowledge of both the displacement and traction fields on $\partial \Omega$. This is a more general inverse problem than we consider here, so it provides uniqueness for our case. This is because here we know the displacement everywhere; hence knowledge of the stiffness tensor locally on a boundary outside of the inhomogeneity enables the traction vector to be calculated there. Furthermore in [31] this proof of uniqueness of the boundary inverse problem is extended for transversely isotropic elastic materials in three-dimensional space, provided that the normal vector of the boundary does not coincide with the principal direction of the transverse anisotropy. This coincides with the results of [35], who show for transversely isotropic elastic materials in three-dimensional space that when the normal vector of the boundary coincides with the principal direction of the transverse anisotropy the object can be illusionarily cloaked. Such a result implies that, if this is the case, the inverse problem can be made nonunique.

The nonlinear inverse problem can be stated mathematically as finding $\nu \in \mathcal{P}$, where $\mathcal{P}$ is the material parameter function space such that the difference equation

$$
F(\nu)=\mathbf{u}(\mathbf{x} ; \nu)-\mathbf{u}^{\text {meas }}(\mathbf{x})=0, \quad \mathbf{x} \in \Omega,
$$

is satisfied. Here $\mathbf{u}$ is the solution of (2.1), $\mathbf{u}^{\text {meas }}$ is the measured displacement field, and $\nu$ are the material functions to be determined. In practice, $\mathbf{u}^{\text {meas }}$ is subject to experimental errors and can be measured only on a finite set, say $\mathcal{M} \in \Omega$. Hence (4.1) is replaced by

$$
\min _{\nu}\|F(\nu)\|, \quad \mathbf{x} \in \mathcal{M},
$$

in a suitable norm. The Newton-Kantorovich method for (4.1) amounts to iteratively solving the following operator equation, for the update function $s$ :

$$
F^{\prime}(\nu) s=\mathbf{u}^{\prime}(\nu) s=-F(\nu),
$$

where $\mathbf{u}^{\prime}(\nu) s$ is the Fréchet derivative of $\mathbf{u}$ with respect to $\nu$. That is, the next update is $\nu+s$. It can be seen that this equation directly utilizes the Fréchet derivative of the map $\mathbb{T}: \nu \rightarrow \mathbf{u}$, which is fundamental to the inverse problem and is analyzed here. We observe that this map $\mathbb{T}$ is defined implicitly by (2.1) or, equivalently, (3.21). If optimization techniques are used for computationally solving the inverse problem, they too need use of the Fréchet derivative of the the map $\mathbb{T}$.

There are many implicit forms for the operator $\mathbb{T}$, and the mapping properties are unique once the function spaces have been fixed. We use integral operators here 
to derive properties of the map through its linearization. It appears that these may give preferable mapping properties to other formulations such as finite element ones as they use stronger function spaces.

In what follows we drop the bar on the material difference functions for simplicity of notation with no misunderstanding.

4.1. Inverse problem for difference stiffness. We are considering here the isotropic case when there are two functions describing the stiffness of the media. For simplicity we consider in this section the case when only one of the Lamé parameters differs, in turn, from the Green state case; we consider the case of both differing in section 4.3. The implicit functional

$$
\boldsymbol{\xi}(\sigma, \mathbf{u})=\mathbf{u}(\sigma, \mathbf{x})+\mathbb{K}_{\sigma} \mathbf{u}(\sigma, \mathbf{x})-\mathbf{u}^{\mathrm{inc}}(\mathbf{x})=0, \quad \sigma=\{\lambda, \mu\},
$$

which is obtained from the integral representation for the direct problem equation (3.15), can be utilized with the implicit function theorem to obtain the Fréchet derivative of the mapping $\mathbb{T}: \sigma \rightarrow \mathbf{u}$. Here in the inverse problem $\mathbf{u}$ is to be measured throughout $\Omega$. First appropriate function spaces for the mapping $\boldsymbol{\xi}$ must be defined, so note $\boldsymbol{\xi}: X^{\sigma} \times \mathrm{H}^{1}(\bar{\Omega})^{3} \rightarrow \mathrm{H}^{0}(\bar{\Omega})^{3}$ with $X^{\sigma}=\left\{\sigma=\{\lambda, \mu\}: \sigma \in \mathrm{H}^{0}(\bar{\Omega}), 0<\sigma<M\right\}$ for some positive real constant $M$.

Lemma 4.1. The map $\mathbb{T}: \sigma \rightarrow \mathbf{u}$ from $X^{\sigma}$ to $H^{0}(\bar{\Omega})^{3}$ with $\sigma$ chosen from $\{\lambda, \mu\}$ is Fréchet differentiable with respect to $\sigma$, with Fréchet differential

$$
\begin{aligned}
& \left(\mathbb{T}^{\prime}(\sigma)\right) s=\mathbf{u}^{\prime}(\sigma) s=-\left[\mathbb{I}+\mathbb{K}_{\sigma}\right]^{-1} \mathbb{K}_{s} \mathbf{u}(\sigma), \\
& \left.\mathbb{T}^{\prime}(\sigma) s\right|_{k}=-\widetilde{\mathbb{K}}_{s} \mathbf{u}=-\int_{\Omega} u_{j, i}\left(\sigma, \mathbf{x}^{\prime}\right) \widetilde{k}_{i j k}^{s}\left(\mathbf{x}, \mathbf{x}^{\prime}\right) s\left(\mathbf{x}^{\prime}\right) d V^{\prime},
\end{aligned}
$$

where the kernels $\widetilde{k}_{i j k}^{s}$ involve $\widetilde{\mathbf{G}}$, which is the Green function pertinent for the stiffness difference $\sigma$ (see (2.14)), and are given by (3.14) with $\widetilde{\mathbf{G}}$ replacing $\mathbf{G}$.

Proof. Observe that the differential $\mathbf{u}^{\prime}(\sigma) s$ defines a linear operator, the Fréchet derivative, with $s \in X$. To prove differentiability of $\mathbb{T}$ we check the conditions of the implicit function theorem (our form is quoted in Appendix C) on the functional $\boldsymbol{\xi}$. Theorem 3.2 assures us that there is only one solution $\mathbf{u}$ in $\mathrm{H}^{0}(\bar{\Omega})^{3}$; then we proceed as follows.

It is straightforward to show that $\boldsymbol{\xi}, \boldsymbol{\xi}_{\sigma}$, and $\boldsymbol{\xi}_{\mathbf{u}}$ are continuous in $\sigma$ and $\mathbf{u}$, where the partial Fréchet derivative of (4.4) with respect to $\sigma$ is given through

$$
\boldsymbol{\xi}_{\sigma}(\sigma, \mathbf{u}) s=\mathbb{K}_{s} \mathbf{u},
$$

because (3.13) is linear in $\sigma$; also note $s \in X^{\sigma}$. Further, the partial Fréchet derivative of $\boldsymbol{\xi}$ with respect to $\mathbf{u}$ is

$$
\boldsymbol{\xi}_{\mathbf{u}}(\sigma, \mathbf{u}) \mathbf{s}=\left(\mathbb{I}+\mathbb{K}_{\sigma}\right) \mathbf{s},
$$

as (4.4) is linear in $\mathbf{u}$, with again $\mathbf{s} \in \mathrm{H}^{1}(\bar{\Omega})^{3}$.

The only further condition necessary for the application of the implicit function theorem is that $\left[\boldsymbol{\xi}_{\mathbf{u}}(\sigma, \mathbf{u})\right]^{-1}$ is bounded, and this has been proven in Theorem 3.2. The explicit expression for the Fréchet derivative is given by the implicit function theorem (4.5a). Equation (4.5b) can be obtained by use of the integral equation satisfied by the Green function $\widetilde{\mathbf{G}}$, namely (2.14). This derivation is quite technical, and we shall just sketch the derivation here. 
First we find it convenient to introduce a further modification of the operator defined in (3.2) by appending a tilde to the operator when the Green displacement tensor in its definition, $\mathbf{G}$, is changed to $\widetilde{\mathbf{G}}$; cf. (2.12) and (2.13). Thus the extended operator described is

$$
\left.\widetilde{\mathbb{K}}_{C} \mathbf{u}\right|_{k}=\int_{\Omega} \widetilde{\Sigma}_{i j k}^{\prime C}\left(\mathbf{x}, \mathbf{x}^{\prime}\right) u_{j, i}\left(\mathbf{x}^{\prime}\right) d V^{\prime}
$$

with $\widetilde{\Sigma}_{i j k}^{\prime C}$ defined through $(2.13)$ as

$$
\widetilde{\Sigma}_{i j k}^{\prime C}\left(\mathbf{x}, \mathbf{x}^{\prime}\right)=C_{i j \ell m}\left(\mathbf{x}^{\prime}\right) \widetilde{G}_{\ell k, m^{\prime}}\left(\mathbf{x}, \mathbf{x}^{\prime}\right) .
$$

We observe that a prime has been used as a superscript on the stress tensor symbol to denote the fact that the derivative is on the second argument of $\widetilde{\mathbf{G}}$ when compared to (2.13). We shall carry out the calculation for the more general case of the stiffness tensor $\mathbf{C}$ rather than just the isotropic case, and in the differential we use the roman symbol s for the increment $\delta \mathbf{C}$ of the stiffness tensor. We observe that the fundamental solution of (2.1) with stiffness tensor $\mathbf{C}(\mathbf{x})$ and with the difference density zero satisfies

$$
\mathbf{G}=\left(\mathbb{I}+\mathbb{K}_{C}\right) \widetilde{\mathbf{G}},
$$

from (2.14). Then on substitution of the first term on the right-hand side of this equation for $\mathbf{G}$ into the representation of $\mathbb{K}_{\mathrm{s}} \mathbf{u}$, we obtain

$$
\int_{\Omega} \mathrm{s}_{i j \ell m}\left(\mathbf{x}^{\prime}\right) \widetilde{G}_{\ell k, m^{\prime}}\left(\mathbf{x}, \mathbf{x}^{\prime}\right) u_{j, i}\left(\mathbf{x}^{\prime}\right) d V^{\prime}=\int_{\Omega} \widetilde{\Sigma}_{i j k}^{\prime \mathrm{s}}\left(\mathbf{x}, \mathbf{x}^{\prime}\right) u_{j, i}\left(\mathbf{x}^{\prime}\right) d V^{\prime}=\left.\widetilde{\mathbb{K}}_{\mathrm{s}} \mathbf{u}\right|_{k},
$$

and furthermore substitution of the second term on the right-hand side of (4.10) for G shows

$$
\int_{\Omega} d V^{\prime} \mathrm{s}_{i j \ell m}\left(\mathbf{x}^{\prime}\right) u_{j, i}\left(\mathbf{x}^{\prime}\right) \partial_{m^{\prime}} \int_{\Omega} \Sigma_{n r k}^{C}\left(\mathbf{x}^{\prime \prime}, \mathbf{x}\right) \widetilde{G}_{r \ell, n^{\prime \prime}}\left(\mathbf{x}^{\prime \prime}, \mathbf{x}^{\prime}\right) d V^{\prime \prime} .
$$

To first proceed formally we take the differentiation inside the integral

$$
\int_{\Omega} d V^{\prime} \mathrm{s}_{i j \ell m}\left(\mathbf{x}^{\prime}\right) u_{j, i}\left(\mathbf{x}^{\prime}\right) \int_{\Omega} \Sigma_{n r k}^{C}\left(\mathbf{x}^{\prime \prime}, \mathbf{x}\right) \widetilde{G}_{r \ell, n^{\prime \prime} m^{\prime}}\left(\mathbf{x}^{\prime \prime}, \mathbf{x}^{\prime}\right) d V^{\prime \prime}
$$

and interchange of order of integration, plus take the $n^{\prime \prime}$-derivative out of the integral, to find

$$
\begin{aligned}
& \int_{\Omega} \Sigma_{n r k}^{C}\left(\mathbf{x}^{\prime \prime}, \mathbf{x}\right) \partial_{n^{\prime \prime}}\left(\int_{\Omega} \mathrm{s}_{i j \ell m}\left(\mathbf{x}^{\prime}\right) u_{j, i}\left(\mathbf{x}^{\prime}\right) \widetilde{G}_{r \ell, m^{\prime}}\left(\mathbf{x}^{\prime \prime}, \mathbf{x}^{\prime}\right) d V^{\prime}\right) d V^{\prime \prime} \\
& =\left.\int_{\Omega} \Sigma_{n r k}^{C}\left(\mathbf{x}^{\prime \prime}, \mathbf{x}\right) \partial_{n}^{\prime \prime} \widetilde{\mathbb{K}}_{\mathrm{s}} \mathbf{u}\left(\mathbf{x}^{\prime \prime}\right)\right|_{r} d V^{\prime \prime} \\
& =\mathbb{K}_{C} \widetilde{\mathbb{K}}_{\mathrm{s}} \mathbf{u}(\mathbf{x}) .
\end{aligned}
$$

In obtaining this result we have used once the interchange of indices symmetry on the two types of Green tensor. It now follows that

$$
\left[\mathbb{I}+\mathbb{K}_{\sigma}\right] \mathbf{u}^{\prime}(\sigma) \mathrm{s}=\left[\mathbb{I}+\mathbb{K}_{\sigma}\right] \widetilde{\mathbb{K}}_{\mathrm{s}} \mathbf{u}(\mathbf{x}),
$$


and we formally have the result. To provide the rigor we observe it is necessary to subtract and add a correction term when moving the derivatives into and out of the above integrals. In particular a correction term over a surface of a sphere surrounding the singularity must be subtracted from the volume integral when we move the derivative $\partial_{m^{\prime}}$ into the weakly singular integral; e.g., see [27, p. 242]. Equally, when we move the derivative $\partial_{s^{\prime \prime}}$ out of the integral, a correction term over a surface of a sphere surrounding the singularity must be added to the volume integral; these two terms can be shown to cancel. The order of integration can be interchanged on the iterated integrals when the correction terms are present because the integrals interpreted as singular integrals are absolutely convergent [27, Chap. IX].

To complete the derivation of $(4.5 \mathrm{~b})$ we first make the observation that (4.8) and (3.13) differ in which type of displacement Green tensor is used in their definitions, so that the kernels are as (3.14) with $\mathbf{G}$ replaced by $\widetilde{\mathbf{G}}$. Then to return to the isotropic case considered here, we note that $\mathrm{s}_{i j \ell m}$ has the same form as the right-hand side of (3.7) (when $\mathbf{C}$ is replaced by s), so that insertion of this form into the right-hand side of (4.8) yields the representation

$$
\widetilde{\mathbb{K}}_{s} \mathbf{u}=\int_{\Omega} s\left(\mathbf{x}^{\prime}\right) u_{j, i}\left(\sigma, \mathbf{x}^{\prime}\right) \widetilde{k}_{i j k}\left(\mathbf{x}, \mathbf{x}^{\prime}\right) d V^{\prime}
$$

We repeat that here the weakly singular kernels $\widetilde{k}_{i j k}$ are given by (3.14) but using $\widetilde{\mathbf{G}}$ instead of $\mathbf{G}$, and $s$ is the update in either $\lambda$ or $\mu$.

We observe from this differential that the update equation for the increment function $s$ in any Newton-Kantorovich scheme requires inversion of the operator in this equation. This means that the update is found through inversion of a first kind integral operator, which are well known to be ill-posed in most cases. The mapping properties of this operator will be discussed further in the next section.

The evaluation of $\widetilde{\mathbf{G}}$ in a computation algorithm is computationally expensive. However, in an electromagnetic version of this inverse problem the equivalent Green function has been used as the basis of an algorithm [41].

4.1.1. Mapping properties of the inverse stiffness map $\mathbb{T}^{-1}$. The Fréchet derivative at a difference stiffness of zero is more simply evaluated than (4.5b). When both terms of $\bar{\sigma}$ are zero the Fréchet differential is given by

$$
\mathbf{u}^{\prime}(\sigma) s=-\widetilde{\mathbb{K}}_{s} \mathbf{u}^{\mathrm{inc}}=-\int_{\Omega} \Sigma_{i j k}^{s}\left(\mathbf{x}^{\prime}, \mathbf{x}\right) u_{j, i}^{\mathrm{inc}}\left(\mathbf{x}^{\prime}\right) d V^{\prime} .
$$

This linearization (also the Born approximation) provides a straightforward method, but is of limited use in attempting solution of the inverse problem by a modified Newton method.

The linearization of the operator $\mathbb{T}$ about $\bar{\sigma}$ equal to zero can now be used to examine the conditioning of the nonlinear operator $\mathbb{T}^{-1}$ near this value of $\sigma$. For simplification of exposition we define the new integral operators $\mathbb{K}^{B}(\cdot)=\mathbb{K}_{(\cdot)} \mathbf{u}^{\text {inc }}$ and $\mathbb{K}^{N K}(\cdot)=\widetilde{\mathbb{K}}_{(\cdot)} \mathbf{u}$. It is easily seen that as $\bar{\sigma} \rightarrow 0$, then $\mathbf{u} \rightarrow \mathbf{u}^{\text {inc }}, \widetilde{\boldsymbol{\Sigma}} \rightarrow \boldsymbol{\Sigma}$, and $\mathbb{K}^{N K} \rightarrow \mathbb{K}^{B}$. The linearization of the operator $\mathbb{T}$ about a difference stiffness of zero can then be used to examine the conditioning of the nonlinear operator $\mathbb{T}^{-1}$ near this value of $\sigma$.

Equation (4.12) provides a definition of the Born operator $\mathbb{K}^{B}: H^{p} \rightarrow H^{q}$, and we wish to determine the values of $p$ and $q$ that determine the regularity of this operator in the scale of Sobolev spaces. The mapping properties of the operator are 
determined by the principal symbol of its kernel, and this is purely a property of the Green tensor $\nabla \mathbf{G}$ near $\mathbf{x}=\mathbf{x}^{\prime}$, which has been calculated in Appendix A. The principal symbol then is the Fourier transform of the appropriate part of $\nabla \mathbf{G}$, which has been shown to be $|\boldsymbol{\xi}|^{-1}$ in (A.11). The kernel given by equation is a smoothing operator, and it follows straightforwardly that $\mathbb{K}^{B}: \mathrm{H}^{0}(\bar{\Omega})^{3} \rightarrow \mathrm{H}^{1}(\bar{\Omega})$ and that the inverse operator to the Born map is equivalent to first order differentiation. This will mean that the operator $\left(\mathbb{K}^{B}\right)^{-1}$ is unbounded and not continuous on function spaces $\mathrm{H}^{0}(\bar{\Omega})$ because $\mathrm{H}^{1}(\bar{\Omega})$ is compactly embedded in $\mathrm{H}^{0}(\bar{\Omega})$. Regularization of this operator, and hence $\mathbb{T}^{-1}$, is necessary to restore continuity for this problem that is of mild ill-posedness. Mollification is one of the several techniques available and has been used for differentiation-type operators in various inverse problems [28, 49, 38].

When we examine the mapping properties of the operator $\mathbb{K}^{N K}$ only the singularities of the kernel need to be taken into consideration. This is seen by forming $\widetilde{\boldsymbol{\Sigma}}-\boldsymbol{\Sigma}$, and it follows because of the linearity of (2.1) that this difference satisfies a homogeneous partial differential equation and, moreover, is in $C^{1}(\Omega)$. This implies that the singular properties of $\widetilde{\boldsymbol{\Sigma}}$ are the same as those of $\boldsymbol{\Sigma}$, and therefore it follows that the function space mapping property of $\mathbb{K}^{N K}$ is identical to $\mathbb{K}^{B}$. We therefore have the following result.

TheOREM 4.2. The linearization of the map $\mathbb{T}^{-1}: \mathbf{u} \rightarrow \sigma$, given by (4.5b), is a continuous map from $H^{1}(\bar{\Omega})^{3}$ into $H^{0}(\bar{\Omega})$; furthermore it is equivalent to a first order differential operator and therefore is an unbounded map from $H^{0}(\bar{\Omega})^{3}$ to $H^{0}(\bar{\Omega})$.

Proof. This follows heuristically from above, and rigorously from the results stated below Corollary 3.3, where it was shown that $\mathbb{K} \mathbf{u}$ mapping $\sigma$ is a compact operator into $\mathrm{H}^{0}(\bar{\Omega})$ and this is also true for $\widetilde{\mathbb{K}} \mathbf{u}$.

4.2. Inverse problem for difference density. The implicit functional

$$
\boldsymbol{\xi}(\rho, \mathbf{u})=\mathbf{u}(\rho ; \mathbf{x})-\mathbb{R}_{\rho} \mathbf{u}(\rho ; \mathbf{x})-\mathbf{u}^{\text {inc }}(\mathbf{x})=0,
$$

which is obtained from the integral representation for the direct problem (3.19), can be utilized with the implicit function theorem to obtain the Fréchet derivative of the mapping $\mathbb{T}: \rho \rightarrow \mathbf{u}$. Here in the inverse problem $\mathbf{u}$ is to be measured throughout $\Omega$. First appropriate function spaces for the mapping $\boldsymbol{\xi}$ must be defined, so note $\boldsymbol{\xi}: X^{\rho} \times \mathrm{H}^{0}(\bar{\Omega})^{3} \rightarrow \mathrm{H}^{0}(\bar{\Omega})^{3}$ with $X^{\rho}=\left\{\rho: \rho \in \mathrm{H}^{0}(\bar{\Omega}), 0<\rho<M\right\}$ for some positive real constant $M$. We can then prove the following result for the partial Fréchet derivative with respect to $\bar{\rho}$.

Lemma 4.3. The map $\mathbb{T}: \rho \rightarrow \mathbf{u}$ from $X^{\rho}$ to $H^{0}(\bar{\Omega})^{3}$ is Fréchet differentiable with respect to $\rho$, with Fréchet differential

$$
\begin{aligned}
\left(\mathbb{T}^{\prime}(\rho)\right) s=\mathbf{u}^{\prime}(\rho) s & =\left[\mathbb{I}-\mathbb{R}_{\rho}\right]^{-1} \mathbb{R}_{s} \mathbf{u}(\rho) \\
& =\omega^{2} \int_{\Omega} \mathbf{u}\left(\rho ; \mathbf{x}^{\prime}\right) \cdot \widetilde{\mathbf{G}}\left(\mathbf{x}, \mathbf{x}^{\prime}\right) s\left(\mathbf{x}^{\prime}\right) d V^{\prime},
\end{aligned}
$$

where $\widetilde{\mathbf{G}}$ is the Green function pertinent for the density difference $\rho$; see (2.12).

Proof. Observe that $\mathbf{u}^{\prime}(\rho)$ is a linear operator, the Fréchet derivative with respect to $s \in X^{\rho}$. To prove differentiability of $\mathbb{T}$ we check the conditions of the implicit function theorem on the functional $\boldsymbol{\xi}$. Theorem 3.4 assures us that there is only one solution $\mathbf{u}$ in $\mathrm{H}^{0}(\bar{\Omega})^{3}$; then we proceed as follows.

It is straightforward to show that $\boldsymbol{\xi}, \boldsymbol{\xi}_{\rho}$, and $\boldsymbol{\xi}_{\mathbf{u}}$ are continuous in $\rho$ and $\mathbf{u}$, where the partial Fréchet derivative of (4.13) with respect to $\rho$ is given through

$$
\boldsymbol{\xi}_{\rho}(\rho, \mathbf{u}) s=-\mathbb{R}_{s} \mathbf{u}
$$


because (3.18) is linear in $\rho$; also note $s \in X^{\rho}$. Further, the partial Fréchet derivative of $\boldsymbol{\xi}$ with respect to $\mathbf{u}$ is

$$
\boldsymbol{\xi}_{\mathbf{u}}(\rho, \mathbf{u}) \mathbf{s}=\left(\mathbb{I}-\mathbb{R}_{\rho}\right) \mathbf{s}
$$

as (4.4) is linear in $\mathbf{u}$, with again $\mathbf{s} \in \mathrm{H}^{0}(\bar{\Omega})^{3}$. The only further condition necessary for the application of the implicit function theorem is that $\left[\boldsymbol{\xi}_{\mathbf{u}}(\rho, \mathbf{u})\right]^{-1}$ be bounded, and this has been proven in Theorem 3.4. The explicit expression for the Fréchet derivative is given by the implicit function theorem as (4.14a).

The more usable form of (4.14b) can be obtained from (4.14a) by use of the integral equation satisfied by the Green function $\widetilde{\mathbf{G}}$, namely (2.14) when $\mathbf{C}=\mathbf{C}^{0}$, so that

$$
\mathbf{G}=\left(\mathbb{I}-\mathbb{R}_{\rho}\right) \widetilde{\mathbf{G}} .
$$

Substitution of the above expression for $\mathbf{G}$ in the kernel of $\mathbb{R}_{s}$ into (4.14a) and interchange of the order of integration between $\mathbb{R}_{s}$ and $\mathbb{R}_{\rho}$, together with the symmetry properties of the Green tensors, yields the alternative form of the differential

$$
u_{\ell}^{\prime}(\rho) s=\omega^{2} \int_{\Omega} u_{j}\left(\rho, \mathbf{x}^{\prime}\right) \widetilde{G}_{j \ell}\left(\mathbf{x}, \mathbf{x}^{\prime}\right) s\left(\mathbf{x}^{\prime}\right) d V^{\prime}=\left.\widetilde{\mathbb{R}}_{s} \mathbf{u}\right|_{\ell},
$$

where we have for future use defined a new integral operator that has been slightly modified from (3.18) by the replacement of $\mathbf{G}$ by $\widetilde{\mathbf{G}}$.

Observe from the differential in (4.14b) that the update equation for the increment function $s$ in any Newton-Kantorovich scheme requires inversion of the operator in this equation. The properties of this Fréchet differential operator will be discussed further in the next section.

4.2.1. Mapping properties of the inverse density map $\mathbb{T}^{-1}$. First note that the Fréchet derivative at a difference density of zero is more simply evaluated than (4.14b). Then the Fréchet differential is given by

$$
u_{k}^{\prime}(\rho) s=\omega^{2} \int_{\Omega} G_{j k}\left(\mathbf{x}, \mathbf{x}^{\prime}\right) u_{j}^{\mathrm{inc}}\left(\mathbf{x}^{\prime}\right) s\left(\mathbf{x}^{\prime}\right) d V^{\prime}=\left.\mathbb{R}_{s} \mathbf{u}^{\mathrm{inc}}\right|_{k} .
$$

This linearization is in fact what is known as the Born approximation, and it provides a straightforward method, but of limited use, for solving the inverse problem by a modified Newton method.

Similar to our discussion in section 4.1.1, (4.17) provides a definition of the Born operator mapping $s$ into $\mathbf{u}$, and we wish to determine the regularity of this operator in the scale of Sobolev spaces. The mapping properties of the operator are determined by the principal symbol of its kernel, and this is purely a property of the Green tensor $\mathbf{G}$ near $\mathbf{x}=\mathbf{x}^{\prime}$, which has been calculated in Appendix A. The principal symbol then is the Fourier transform of the appropriate part of $\mathbf{G}$, which has been shown to be $|\boldsymbol{\xi}|^{-2}$ in (A.10). The kernel given by equation is a smoothing operator, and it follows straightforwardly that $\mathbb{R}^{B}: \mathrm{H}^{0}(\bar{\Omega})^{3} \rightarrow \mathrm{H}^{2}(\bar{\Omega})$ and that the inverse operator to the Born map is equivalent to second order differentiation.

When we examine the mapping properties of the operator $\mathbb{R}_{s} \mathbf{u}$, which is the Newton-Kantorovich map for the derivative of $\mathbb{T}$ linearized about an arbitrary difference density, we see by an argument similar to that used in section 4.1.1 that the mapping properties are the same as those of the Born map. We therefore have the next result. 
TheOREM 4.4. The linearization of the map $\mathbb{T}^{-1}: \mathbf{u} \rightarrow \rho$ is a continuous map from $H^{2}(\bar{\Omega})^{3}$ into $H^{0}(\bar{\Omega})$ and is therefore equivalent to a second order differential operator. Furthermore it is an unbounded map from $H^{0}(\bar{\Omega})^{3}$ to $H^{0}(\bar{\Omega})$.

Proof. This follows heuristically from the above, and rigorously from the results stated below Theorem 3.4, where it was shown that $\mathbb{R} \mathbf{u}$ mapping $\rho$ is a compact operator into $\mathrm{H}^{0}(\bar{\Omega})$ and this is also true for $\widetilde{\mathbb{R}} \mathbf{u}$.

4.3. Inverse problem for multiple differences. We consider here the inverse problem of determination of difference stiffness tensor and density. The map is $\mathbb{T}$ : $\nu \rightarrow \mathbf{u}$ from $X=\left\{X^{\rho} \times X^{\lambda} \times X^{\mu}\right\}$ into $\mathrm{H}^{0}(\bar{\Omega})^{3}$, where $\nu \in(\lambda, \mu, \rho)$. We now denote each of the linearizations with respect to $\rho, \lambda$, and $\mu$ as $\mathbb{T}_{\rho}, \mathbb{T}_{\lambda}$, and $\mathbb{T}_{\mu}$, respectively; we see that the gradient of the map $\mathbb{T}: \nu \rightarrow u$ from $X$ into $\mathrm{H}^{0}(\bar{\Omega})^{3}$ is given by

$$
\left(\mathbb{T}^{\prime}(\rho)\right) s=\mathbf{u}^{\prime}(\rho) s=\left[\begin{array}{lll}
\mathbb{T}_{\rho} & \mathbb{T}_{\lambda} & \mathbb{T}_{\mu}
\end{array}\right] \mathbf{s},
$$

where $\mathbf{s}=\left[\begin{array}{lll}s_{\rho} & s_{\lambda} & s_{\mu}\end{array}\right]^{T}$ is a three-vector in $X$. The appropriate expressions for the partial Fréchet derivatives in the three-vector gradient operator are given by the results in Lemmata 4.1 and 4.3.

Prior to discussing the mapping properties of the full Fréchet differential of $\mathbb{T}$, we provide the proof that (4.18) provides the linearization of the map $\mathbb{T}$.

TheOREM 4.5. The map $\mathbb{T}: \nu \rightarrow \mathbf{u}$ from $X$ into $H^{0}(\bar{\Omega})^{3}$, for an isotropic stiffness, is Fréchet differentiable with respect to $\nu$, with a Fréchet differential given by

$$
\left(\mathbb{T}^{\prime}(\rho)\right) s=-\left[\mathbb{I}-\mathbb{R}_{\rho}+\mathbb{K}_{\lambda}+\mathbb{K}_{\mu}\right]^{-1}\left(-\mathbb{R}_{s_{\rho}}+\mathbb{K}_{s_{\lambda}}+\mathbb{K}_{s_{\mu}}\right),
$$

and has gradient representation (4.18).

Proof. The implicit functional

$$
\boldsymbol{\xi}(\nu, \mathbf{u})=\mathbf{u}(\nu ; \mathbf{x})-\mathbb{R}_{\rho} \mathbf{u}(\nu ; \mathbf{x})+\mathbb{K}_{\lambda} \mathbf{u}(\nu, \mathbf{x})+\mathbb{K}_{\mu} \mathbf{u}(\nu, \mathbf{x})-\mathbf{u}^{\text {inc }}(\mathbf{x})=0
$$

is used with the implicit function theorem in proving this theorem. Standard analysis similar to that provided earlier for the partial Fréchet derivatives shows this result. The only major difference in the condition necessary for the application of the implicit function theorem is to show that $\left[\boldsymbol{\xi}_{\mathbf{u}}(\nu, \mathbf{u})\right]^{-1}$ is bounded, and this was proven in Corollary 3.6.

The explicit expression for the Fréchet derivative is given by the implicit function theorem as (4.19). However, the more useful form is provided by the gradient of $\mathbb{T}$ in (4.18).

We have now completed examination of the operator $\mathbb{T}$ which maps the material functions into the displacement field. We have shown that this nonlinear mapping is bounded by Corollary 3.6 and that it is differentiable in appropriate function spaces in Theorem 4.5. Furthermore, we have shown that its linearization $\mathbb{T}^{\prime}$ is a compact operator, which implies that $\mathbb{T}^{\prime-1}$ is unbounded. ${ }^{1}$

4.3.1. Mapping properties of the inverse map $\mathbb{T}^{-1}$. The ill-posed part of the map $T^{-1}$ associated with $\mathbb{R}_{\rho}$ makes this full linearization ill-posed and equivalent to second order differentiation for density reconstruction and first order differentiation for imaging of both the shear modulus and the $\lambda$ functions.

\footnotetext{
${ }^{1}$ We observe that this does not imply that $\mathbb{T}$ is necessarily compact in those spaces.
} 
5. Discussion. In the last section we derived rigorous expressions for the partial Fréchet derivatives of the nonlinear operator $\mathbb{T}$ with respect to $\nu$.

We have examined the mapping properties of $\mathbb{T}$ with respect to $\sigma$ as discussed in section 4.1 .1 and given by (4.5b). The kernel in $\widetilde{\mathbb{K}}_{s} \mathbf{u}$, namely $u_{j, i} \widetilde{k}_{i j k}$, determines how a variation $s$ in $\sigma$ propagates its effect by changing the displacement field throughout $\Omega$. This has been shown to be equivalent to an integration which means that the inverse problem is mildly ill-conditioned. This means the reconstruction of $\sigma$ from measurement of the displacement field is not so ill-posed as the density inverse problem which is equivalent to second order differentiation. There are many approaches to the determination of the Lamé parameters in isotropic elasticity, although few of them use the integral approach discussed here; we list a few $[9,12]$. The mapping properties of the linearization in the standard techniques currently used to solve the elastography problem, namely finite elements, are unknown. It appears that linearization based on the singular map (3.8) could lead to better conditioning than the map considered here, but this is as yet undetermined.

For linear integral operator equations that are equivalent to first and second order differentiation, singular function decomposition shows that the inverses of such operators have condition numbers that increase as $n$ and $n^{2}$, respectively, where $n$ is the number of singular functions [17, Chap. 15]. We can use this to make the following observations about discrete versions of the our inverse problems. For the density reconstruction only problem, if we attempt to reconstruct $10^{2}$ pixels along one dimension, i.e., $10^{6}$ in the cube, this will mean that the matrix to be inverted will have a condition number on the order of $10^{12}$, quite ill-conditioned. For the similar problem of just stiffness reconstruction, of either $\lambda$ or $\mu$, the condition number will be $10^{6}$, not well-conditioned but considerably better than for the density case. This simple argument has implications on the image resolution possible in MRE.

When the full problem of elastic imaging is considered as in section 4.3 it is seen that the inverse problem is ill-posed and dominated by the density reconstruction. However, the analysis here has shown that a mechanism of alleviating this is to attempt a coarser reconstruction for the density than the stiffness parameters. Hence an appropriate regularization would restore continuity of the solution to the measured data but still achieve relevantly high resolution of the stiffness functions. It is important to appreciate that the degree of ill-conditioning determines the possible resolution of the reconstructed image.

In conclusion we have developed a rigorous theory of MRE. The simplistic approach is to just differentiate the displacement field and assume that the resultant strain field is explicitly related to the stiffness of the material, thus yielding a strain image [11], but this is not always a realizable stiffness [6]. Our theory illustrates for just the stiffness case that this is justified in a crude manner, but methods such as those developed here offer better reconstruction. In a sense this is similar to the difference in x-ray imaging between the full theory of CAT and shadow-grams.

The choice of elastic properties for imaging in elastography research remains an open question at this point; the use of the analytical methods described here will help to predict and understand the value and reliability of different parameterizations of elasticity imaging. Furthermore, our results indicate that significant work needs to be done to achieve effective multiparameter reconstructive imaging. We are currently extending this work to include the full elastic imaging problem, incorporating boundary conditions and incompressible elasticity. By also incorporating nonisotropy and viscoelasticity, the analysis will get closer to the real problem as studied by practitioners. 
Appendix A. Singularity of Green tensors. Here we consider the nature of the singularity at the pole of the various Green functions and their derivatives. This is central to our work in discussing the nature of the various integral operators. The solution of (2.3) is important for analysis of the singular behavior of the kernels in various integrals.

A.1. Static Green tensor. The solution of (2.3) when $\omega=0$ is the most important part of this singularity, and for convenience of notation and without loss of generality, we move the origin of coordinates to $\mathbf{x}^{\prime}$, then $R=r$, and we find that the solution of this equation is the symmetric Somiglian's tensor,

$$
\Gamma_{i j}^{0}=\frac{1}{8 \pi \mu(\lambda+2 \mu)}\left((\lambda+3 \mu) \frac{\delta_{i j}}{r}+(\lambda+\mu) \frac{x_{i} x_{j}}{r^{3}}\right) .
$$

The gradient of this function then follows as

$$
\Gamma_{i j, k}^{0}=\frac{1}{8 \pi \mu(\lambda+2 \mu)}\left(-(\lambda+3 \mu) \frac{\delta_{i j} x_{k}}{r^{3}}+\frac{(\lambda+\mu)}{r^{3}}\left[\delta_{i k} x_{j}+\delta_{j k} x_{i}-\frac{3 x_{i} x_{j} x_{k}}{r^{2}}\right]\right),
$$

so that the divergence is

$$
\Gamma_{i j, i}^{0}=\frac{-2 \mu}{8 \pi \mu(\lambda+2 \mu)} \frac{x_{j}}{r^{3}} .
$$

This means that the singular symmetric tensor kernels appearing in (3.10), namely in $S_{k j}^{\lambda}$ from the last formula, become

$$
\Gamma_{i j, i k}^{0}=\frac{2 \mu}{8 \pi \mu(\lambda+2 \mu)}\left\{\frac{3 x_{j} x_{k}}{r^{5}}-\frac{\delta_{j k}}{r^{3}}\right\},
$$

and in $S_{k j}^{\mu}$ it is $\Gamma_{i j, k i}^{0}=\Gamma_{i j, i k}^{0}$, and so

$$
\Gamma_{i j, k k}^{0}=\frac{2(\lambda+\mu)}{8 \pi \mu(\lambda+2 \mu)}\left\{\frac{\delta_{i j}}{r^{3}}-\frac{3 x_{j} x_{i}}{r^{5}}\right\}=-\frac{(\lambda+\mu)}{\mu} \Gamma_{k j, k i}^{0} .
$$

A.2. Harmonic Green tensor. First observe that the fundamental Green function we have to work with is $g^{k}=e^{i k R} / 4 \pi R$, where $R=\left|\mathbf{x}-\mathbf{x}^{\prime}\right|$. For convenience of notation and without loss of generality, we move the origin of coordinates to $\mathbf{x}^{\prime}$, then $R=r$. The singularity of $G$ as $r \rightarrow 0$ is delicate because of the cancellation in the second term in (2.6), and it can be shown that

$$
\begin{aligned}
\lim _{r \rightarrow 0} G_{i j} & =\frac{1}{8 \pi \mu(\lambda+2 \mu)}\left[(\lambda+3 \mu) \frac{\delta_{i j}}{r}+(\lambda+\mu) \frac{x_{i} x_{j}}{r^{3}}\right]+c(\omega, \lambda, \mu) \mathcal{O}(1) \\
& =\Gamma_{i j}^{0}+c(\omega, \lambda, \mu) \mathcal{O}(1) .
\end{aligned}
$$

The gradient and second derivative of this tensor function then yield

$$
\lim _{r \rightarrow 0} G_{i j, k}=\Gamma_{i j, k}^{0}+\mathcal{O}(1), \quad \lim _{r \rightarrow 0} G_{i j, k \ell}=\Gamma_{i j, k \ell}^{0}+\mathcal{O}(1),
$$

so that the divergence is

$$
G_{i j, i}=\Gamma_{i j, i}^{0}+\mathcal{O}(1) \quad \text { or } \quad G_{i j, i}=-\frac{1}{\rho^{0} \omega^{2} 4 \pi} \frac{x_{j} k_{P}^{2}}{r^{3}}+\mathcal{O}(1)
$$


These calculations imply that $\mathbf{G}$ is $\mathcal{O}\left(r^{-1}\right)$; i.e., the singularity is of order $1, \nabla \mathbf{G}$ is $\mathcal{O}\left(r^{-2}\right)$, and $\nabla \nabla \mathbf{G} \rightarrow \mathcal{O}\left(r^{-3}\right)$. Now if we define $\bar{G}=G-\Gamma^{0}$, then it is seen from the above calculations that this function $\bar{G}$ is more regular than $G$. We now need to calculate stress tensor $\Sigma_{i j k}^{C}$ as $r \rightarrow 0$, which is defined in (2.4) but has the stiffness as (2.11). This tensor can be shown to be

$$
\begin{aligned}
\lim _{r \rightarrow 0} \Sigma_{i j k}^{C}= & \bar{\lambda} \delta_{i j} \Gamma_{\ell k, \ell}^{0}+\bar{\mu}\left(\Gamma_{i k, j}^{0}+\Gamma_{j k, i}^{0}\right)+\mathcal{O}(1) \\
= & \frac{1}{8 \pi(\lambda+2 \mu)} \frac{2}{r^{3}}\left(\delta_{i j} x_{k}[-\bar{\lambda}+\tilde{\mu}(\lambda+\mu)]-\tilde{\mu}\left[\mu\left(\delta_{j k} x_{i}+\delta_{i k} x_{j}\right)\right.\right. \\
& \left.\left.+\frac{3 x_{i} x_{j} x_{k}}{r^{2}}(\lambda+\mu)\right]\right)+\mathcal{O}(1) .
\end{aligned}
$$

In a similar manner we have

$$
\lim _{r \rightarrow 0} \Sigma_{i j k}^{C}=\frac{1}{8 \pi(\lambda+2 \mu)} \frac{2}{r^{3}}\left[\mu \delta_{i j} x_{k}-\mu\left(\delta_{j k} x_{i}+\delta_{i k} x_{j}\right)-\frac{3 x_{i} x_{j} x_{k}}{r^{2}}(\lambda+\mu)\right]+\mathcal{O}(1),
$$

and so we finally see that $\Sigma \rightarrow \mathcal{O}\left(r^{-2}\right)$ and $\nabla \boldsymbol{\Sigma} \rightarrow \mathcal{O}\left(r^{-3}\right)$.

A.3. Time dependent heterogeneous Green tensor. This tensor is defined through (2.12) and (2.13). The behavior as $\mathbf{x} \rightarrow \mathbf{x}^{\prime}$ for these tensors is more complicated than for those discussed in the last section. However, provided the $\nu(\mathbf{x})$ are all continuous functions, then by subtraction of (2.3) from (2.12) it can be shown that the singularities are of the same order as the homogeneous time dependent ones.

A.4. Stress tensor surface integrals. We first consider here the integral formed by subtraction of the sphere of radius $\epsilon$ about the point $\mathbf{x}^{\prime}$ in (2.9) in order to provide evaluation of the singular integrals, namely

$\lim _{\epsilon \rightarrow 0} \int_{S_{\epsilon}}\left[n_{i} \Sigma_{i j k}\left(\mathbf{x}, \mathbf{x}^{\prime}\right) u_{j}(\mathbf{x})-n_{i} \tau_{i j}(\mathbf{x}) G_{j k}\left(\mathbf{x}, \mathbf{x}^{\prime}\right)\right] d S=\lim _{\epsilon \rightarrow 0} \int_{S_{\epsilon}}\left[n_{i} \Sigma_{i j k}\left(\mathbf{x}, \mathbf{x}^{\prime}\right) u_{j}(\mathbf{x})\right] d S$,

as $\mathbf{G}=\mathcal{O}(1 / r)$ and $\boldsymbol{\tau}$ is bounded. So we examine the integral on the right-hand side, but for generality we replace the $\Sigma_{i j k}^{C^{0}}$ by $\Sigma_{i j k}^{C}$ and then, using (A.6), show that this integral becomes

$$
\begin{aligned}
& \frac{-2}{8 \pi(\lambda+2 \mu)}\left\{\int_{S_{\epsilon}} d \Omega \frac{x_{j} x_{k}}{\epsilon^{2}} u_{j}\left[\left(-\bar{\lambda}+\frac{\bar{\mu}}{\mu}(\lambda+\mu)\right)-\frac{\bar{\mu}}{\mu}[\mu+3(\lambda+\mu)]-\int_{S_{\epsilon}} d \Omega u_{k}\right\}\right. \\
& \text { (A.8) }=\frac{u_{k}\left(\mathbf{x}^{\prime}\right)}{3(\lambda+2 \mu)}\left[\bar{\lambda}+2 \frac{\bar{\mu}}{\mu}(\lambda+3 \mu)\right],
\end{aligned}
$$

where $\bar{\lambda}, \bar{\mu}$ correspond to the difference stiffness parameters. Observe that when $\bar{\mu}=\mu$ and $\bar{\lambda}=\lambda$ the integral in (A.8) becomes $u_{k}\left(\mathbf{x}^{\prime}\right)$, as used in (2.10). When $\bar{\mu}=\bar{\lambda}=0$ the integral is zero.

When we use the above equations for an isotropic medium in the expression for $\alpha$ in (3.3) it can be seen that

$$
\begin{aligned}
\alpha & =1+\frac{1}{u_{j}(\mathbf{x})} \int_{S_{\epsilon_{x}^{\prime}}} n_{i} \Sigma_{i j k}^{C}\left(\mathbf{x}, \mathbf{x}^{\prime}\right) u_{j}\left(\mathbf{x}^{\prime}\right) d S^{\prime} \\
& =\frac{2(\bar{\mu}+\mu)}{3 \mu}+\frac{(\bar{\lambda}+\lambda)+2(\bar{\mu}+\mu)}{3(\lambda+2 \mu)},
\end{aligned}
$$

Copyright (c) by SIAM. Unauthorized reproduction of this article is prohibited. 
where $S_{\epsilon_{x}^{\prime}}$ is a sphere of radius $\epsilon$ centered at $\mathbf{x}^{\prime}$ and $n_{i}$ is the $i$ th component of the unit normal to $S_{\epsilon}$.

A.5. Symbols of Green tensors. The existence theory for the singular integral operators uses the symbol of the integral operator, which is given by the Fourier transform of the kernel, and we list the dominant part of the Green tensors here. We need the symbol of the integral operators defined in section 3.1.1, and we evaluate them here. We should first note that the integral operators are defined over $\Omega$ not $\mathbb{R}^{3}$, but this can be easily achieved by the standard trick of extending the domain of definition of the operand of the integral operator by zero on $\mathbb{R}^{3} \backslash \Omega$, and then the symbol of the operator is formed by the Fourier transform of the principal singular part of the kernel. It is the principal singular part of the kernel that determines the symbol because it is that bit that determines the mapping properties of the integral operator, with the lower order terms constituting a compact mapping in the Sobolev space in which the symbol acts. Fundamental to the calculation of the symbols is the knowledge of the Fourier transform of $\Gamma^{0}$, and by elementary means this can be shown to be

$$
{\widehat{\Gamma^{0}}}_{i j}=\frac{1}{\mu(\lambda+2 \mu)}\left(\frac{(\lambda+2 \mu) \delta_{i j}|\boldsymbol{\xi}|^{2}-(\lambda+\mu) \xi_{i} \xi_{j}}{|\boldsymbol{\xi}|^{4}}\right)
$$

where the Fourier transform is denoted by a hat. It is then easily found that $\widehat{\Gamma}_{i j, k}=$ $i \xi_{k} \widehat{\Gamma}_{i j}$ and

$$
\widehat{\Gamma}_{i j, i}=\frac{1}{(\lambda+2 \mu)} \frac{i \xi_{j}}{|\boldsymbol{\xi}|^{2}}
$$

The principal part is therefore

$$
\begin{aligned}
\left.\operatorname{sym} \mathbb{S}_{\lambda}\right|_{i j} & =\text { principal part of } \widehat{S}_{k j}^{\lambda}=\mathcal{F}\left(\Gamma_{\ell j, \ell k}^{0}\right)=\frac{-1}{(\lambda+2 \mu)} \frac{\xi_{j} \xi_{k}}{|\boldsymbol{\xi}|^{2}} \\
\left.\operatorname{sym} \mathbb{S}_{\mu}\right|_{k j} & =\text { principal part of } \widehat{S_{k j}^{\mu}}=\mathcal{F}\left(\Gamma_{i k, j i}^{0}+\Gamma_{j k, i i}^{0}\right) \\
& =\frac{-\lambda}{\mu(\lambda+2 \mu)} \frac{\xi_{j} \xi_{k}}{|\boldsymbol{\xi}|^{2}} .
\end{aligned}
$$

\section{Appendix B. Proof of existence theorems for the direct problems.}

B.1. Proof of Theorem 3.2. To prove this result we will have to utilize singular integral equation theory [27], [19, Chap. IV], [52, Chap. IX] or more generally pseudodifferential operator theory $[43,8]$. This theory involves use of Fourier analysis, and we shall sketch the proof.

The mapping properties of the integral operators that we are studying are determined by the principal singular part of the kernel [43]. Moreover, for such operators the principal symbol is determined from the Fourier transform of the most singular part of the kernel of the integral operator, and this ensures that the principal symbol contains the least negative power (or most positive power) of $|\boldsymbol{\xi}|$. This is because it is this part of the the symbol that determines the mapping properties of the integral operator in the scale of Sobolev spaces [43]. The lower order terms in the remaining part of the symbol constitute a compact mapping in the Sobolev space in which the symbol acts. 
We now consider the principal part of the integral operator $\mathbb{S}_{\bar{\sigma}}$, which we define as $A_{0}$ and write as

$$
\left.A_{0} \mathbf{u}\right|_{k}=\int_{\Omega} S_{k j}^{\bar{\sigma}}\left(\mathbf{x}-\mathbf{x}^{\prime}\right) u_{j}\left(\mathbf{x}^{\prime}\right) \bar{\sigma}\left(\mathbf{x}^{\prime}\right) d V^{\prime}, \quad \bar{\sigma}=\{\lambda, \mu\}
$$

where from Appendix A and (3.10) it follows that $S_{k j}^{\lambda} \rightarrow \Gamma_{\ell k, \ell j}^{0}\left(\mathbf{x}-\mathbf{x}^{\prime}\right)$ and $S_{k j}^{\mu} \rightarrow$ $\left(\Gamma_{i k, j i}^{0}+\Gamma_{j k, i i}^{0}\right)$. Hence $\mathbb{S}_{\bar{\sigma}}=A_{0}+T$, where $T$ is a compact operator in the space $\mathrm{H}^{0}(\bar{\Omega})^{3}$. It is now convenient to remove the integrand term $\bar{\sigma}\left(\mathbf{x}^{\prime}\right)$ from the integral (B.1), so we redefine $\mathbf{u}=\mathbf{u} \bar{\sigma}$ with $\bar{\sigma}>0$, and this modifies the first term in (3.11) to $\gamma_{\sigma}=\alpha / \bar{\sigma}$. It now follows that the principal matrix-valued symbol of the vector-valued operator defined in (3.11), with the modified (B.1), is

$$
\Phi_{i j}^{\sigma}=\gamma_{\sigma}(\mathbf{x}) \delta_{i j}-\left.\operatorname{sym} A_{0}\right|_{i j}(\theta),
$$

where $\theta=\xi /|\xi|$. As $A_{0}$ corresponds to a singular integral operator of form (B.1), its symbol is of order 0 , homogeneous in $\theta$, and it is one of the Fourier transforms listed in (A.12). Furthermore, what is important to the development is that the determinant of $\boldsymbol{\Phi}^{\sigma}$ is positive, and it can be shown to be

$$
\operatorname{det} \boldsymbol{\Phi}^{\sigma}=\gamma_{\sigma}^{2}\left(\gamma_{\sigma}+|\boldsymbol{\xi}|^{2}\right)>|\boldsymbol{\xi}|>0 \quad \forall|\boldsymbol{\xi}|
$$

where $\gamma_{\sigma}>0$ as is seen from the definition of $\gamma$ and (A.9). From this result it follows that the integral equation (3.11) admits a two-sided regularizer (see [27, Chap. IX]) with symbol $\left[\boldsymbol{\Phi}^{\sigma}\right]^{-1}$. To see this consider the singular operator $B_{0}$ formed with the symbol $\left[\boldsymbol{\Phi}^{\sigma}\right]^{-1}$; then by the multiplication rule for symbols [27, Chap. IX, sect. 7], the symbols of $A_{0} B_{0}$ and $B_{0} A_{0}$ are 1 , which implies $A_{0} B_{0}=I+T_{1}$ and $B_{0} A_{0}=I+T_{2}$, where $I$ is the unit operator and $T_{1}$ and $T_{2}$ are compact operators. Furthermore, as the symbol of $A_{0}$ is real, its adjoint operator has the same symbol. These results are true when the symbol of the full operator of $\mathbb{S}_{\bar{\sigma}}$ is used.

The two-sided regularization property implies that the operator (3.11) is a Fredholm operator and is normally solvable by Noether and Atkinson theorems. As the matrix-valued symbol $\Phi$ is symmetric the operator $A_{0}$ has zero index [27, Chap. XIV, sect. 3], [52, Chap. IX, sect. 6]. The regularizer $\left[\boldsymbol{\Phi}^{\sigma}\right]^{-1}$ and its adjoint are equivalent regularizers, and therefore the index of the Fredholm operator is zero, as the dimension of the deficit and the nullity are equal. We observe that the operator $A_{0}$ which corresponds to the static problem has a six-dimensional kernel; it is formed by the possible translations and rotations with basis $\left\{\hat{\mathbf{e}}_{i}, \hat{\mathbf{e}}_{i} \times \hat{\mathbf{r}}\right\}, i \in\{1,2,3\}$ [34]. But dynamic equilibrium of the measurement apparatus will require that any applied forces ensure that these possible null space solutions are not present. It should be remarked that the operator corresponding to the principal symbol used above differs from the operator $\mathbb{S}_{\bar{\sigma}}$ by a compact operator $\left(\right.$ on $\left.\mathrm{H}^{0}(\bar{\Omega})^{3}\right)$. This implies that standard Riesz-Schauder theory can be applied to the regularized form found from $\mathbb{S}_{\bar{\sigma}}$ by use of $B_{0}$. This means that if (3.11) has a unique solution, and therefore the kernel of the operator is zero, the theorem is proved; Lemma 3.1 provides this fact.

As we have previously stated, the mapping properties of the integral operator (B.1) are determined by its symbol. It is seen that the symbol is of order zero and is a $C^{\infty}$-function; hence, by [27, Chap. IX], $\mathbb{S}_{\bar{\sigma}}: \mathrm{H}^{p}(\bar{\Omega})^{3} \rightarrow \mathrm{H}^{p}(\bar{\Omega})^{3}$ is a bounded operator for some $p$; furthermore, from the above theory, the inverse operator $\left(\alpha \mathbb{I}-\mathbb{S}_{\bar{\sigma}}\right)^{-1}$ maps as a bounded operator onto the same spaces. 
B.2. Proof of Theorem 3.4. The existence of the solution for (3.19) follows from standard Riesz-Schauder theory. To apply this theory the compactness of $\mathbb{R}_{\rho}$ : $\mathrm{H}^{0}(\bar{\Omega})^{3} \rightarrow \mathrm{H}^{0}(\bar{\Omega})^{3}$ is required. First consider $\mathbb{R}$, which is an integral operator with a weak singularity, of order 1 (see (A.5)); it follows that $\mathbb{R}:\left(L^{6 / 5}\right)^{3} \rightarrow\left(L^{2}\right)^{3}$ is bounded [19, Chap. IV]. Then as the imbedding $\mathbb{I}: L^{2} \rightarrow L^{6 / 5}$ for $\bar{\Omega}$ is compact $^{2}$ and the composition of a compact and a bounded operator is compact, namely $\mathbb{R} \circ \mathbb{I}$, we have the first compactness. Now consider the operator $B_{\rho}=\bar{\rho} \mathbf{u}, \bar{\rho} \in X^{\rho}$ with $B_{\rho}: \mathrm{H}^{0}(\bar{\Omega})^{3} \rightarrow \mathrm{H}^{0}(\bar{\Omega})^{3}$; then it follows that $B_{\rho}$ is a bounded operator, and again as the composition of a compact and a bounded operator is compact, it follows that $\mathbb{R}_{\rho}$ is a compact operator.

To complete the proof, the Fredholm alternative theorem shows that uniqueness implies existence, and the required uniqueness is given by Lemma 3.1. Therefore we have boundedness of $\left(\mathbb{I}-\mathbb{R}_{\rho}\right)^{-1}$, which will be required when discussing the inverse problem.

B.3. Proof of Theorem 3.5. The symbol of the singular integral operator defined in (3.21) is

$$
\Phi_{i j}=\left(\alpha(\mathbf{x}) \delta_{i j}+\operatorname{sym}\left(\text { principal part }\left.\mathbb{S}_{\bar{\lambda}}\right|_{i j}(\theta)\right)+\operatorname{sym}\left(\text { principal part }\left.\mathbb{S}_{\bar{\mu}}\right|_{i j}(\theta)\right),\right.
$$

where $\theta=\xi /|\xi|$ and the symbol of the principal part of $\mathbb{S}_{\bar{\sigma}}$ is one of the Fourier transforms listed in (A.12). We see from (B.4) that the symbol is of order 0 and is homogeneous in $\theta$. Furthermore, what is important is the determinant of $\boldsymbol{\Phi}^{\sigma}$, which can be shown to be

$$
\operatorname{det} \boldsymbol{\Phi}=\alpha^{2}\left(\alpha+|\xi|^{2}\right)>0 \quad \forall|\boldsymbol{\xi}| .
$$

It follows immediately that the integral equation (3.21) admits a two-sided regularizer (see $\left[27\right.$, Chap. IX]) with symbol $\left[\boldsymbol{\Phi}^{\sigma}\right]^{-1}$. The regularization property with the symbol given in (B.4) implies as previously that the operator (B.1) is a Fredholm operator; it is normally solvable and has index zero.

This implies once again that standard Riesz-Schauder theory can be applied to the regularized form of the operator equation. This means that, provided (3.21) has a unique solution and therefore the kernel of the operator is zero, the theorem is proved; Lemma 3.1 provides this fact.

\section{Appendix C. Implicit function theorem.}

TheOrem C.1. Consider the functional $\xi(v, y), v \in X, y \in Y$, where $\xi: X \times Y \mapsto$ $W$ with $X, Y, W$ being Banach spaces. Then suppose that there exists an open subset $X_{0} \subset X$ such that for every $v \in X_{0}$ the equation $\xi(v, y)=0$ has a unique solution $y=y(v)$ in $Y$. We then have the following:

1. The map $v \rightarrow y(v), v \in X_{0}, y \in Y$, defined from $X_{0} \rightarrow Y$, is continuous upon satisfaction of the additional assumptions

(a) $\xi(v, y)$ is continuous in $v$ and $y$,

(b) $\xi_{y}(v, y)$ is continuous in $v$ and $y$,

(c) $\left[\xi_{y}(v, y)\right]^{-1}$ exists as a bounded mapping $W \rightarrow Y$.

2. Moreover, the map is Fréchet differentiable if the conditions in 1 are satisfied and also $\xi_{v}(v, y)$ is continuous in $v$ and $y$, with

$$
y^{\prime}(v)=-\left[\xi_{y}(v, y)\right]^{-1} \xi_{v}(v, y) .
$$

\footnotetext{
${ }^{2}$ By the Rellich-Kondrachov theorem with $\Omega$ bounded and satisfying the cone property (see [1, Chap. VI]).
} 
Observe that this is the strong form of the implicit function theorem in that the Fréchet derivative is given at all $v \in X_{0}$; the basic form of the implicit function theorem provides information only at a point $v_{0} \in X_{0}$; however, uniqueness of the solution then need not be known a priori.

Acknowledgments. The authors thank the referees for helpful criticisms. We also acknowledge Drs. Marco Gruwel, Peter Latta, and Uta Sboto-Frankenstein, from the NRC Institute for Biodiagnostics in Winnipeg Canada, as well as Mr. Andrei Petrov, from Mechanical Engineering at the University of Canterbury, for providing the data used in Figure 1.

\section{REFERENCES}

[1] R. Adams, Sobolev Spaces, Academic Press, New York, 1975.

[2] H. Ammari, P. Garapon, and F. Jouve, Separation of scales in elasticity imaging: A numerical study, J. Comput. Math., 28 (2010), pp. 354-370.

[3] H. Ammari, P. Garapon, H. Kang, and H. Lee, A method of biological tissues elasticity reconstruction using magnetic resonance elastography measurements, Quart. Appl. Math., 66 (2008), pp. 139-176.

[4] P. Asbach, D. Klatt, B. Schlosser, M. Biermer, M. Muche, A. Rieger, C. LoddenkemPer, R. Somasundaram, T. Berg, B. Hamm, J. Braunand, and I. Sack, Viscoelasticitybased staging of hepatic fibrosis with multifrequency MR elastography, Radiology, 1 (2010), pp. 80-86.

[5] S. Avril, J. M. Huntley, F. Pierron, And D. D. Steele, Heterogeneous stiffness reconstruction using MRI and the virtual fields method, Exper. Mech., 48 (2008), pp. 479-494.

[6] P. E. Barbone And J. C. BAmber, Quantitative elasticity imaging: What can and cannot be inferred from strain images, Phys. Med. Biol., 47 (2002), pp. 2147-2164.

[7] P. E. Barbone And N. H. Gokhale, Elastic modulus imaging: On the uniqueness and nonuniqueness of the elastography inverse problem in two dimensions, Inverse Problems, 20 (2004), pp. 283-296.

[8] J. Chazarain And A. Piriou, Introduction to the Theory of Linear Partial Differential Equations, North-Holland, Amsterdam, 1982.

[9] J. Chen And M. S. Gockenbach, A variational method for recovering planar Lamé moduli, Math. Mech. Solids, 7 (2002), pp. 191-202.

[10] T. J. Connolly and D. J. N. Wall, On Fréchet differentiability of some non-linear operators occurring in inverse problems; An implicit function theorem approach, Inverse Problems, 6 (1990), pp. 949-966.

[11] M. M. Doyley, S. Srinivasan, S. Pendergrass, Z. Wu, and J. Ophir, Comparative evaluation of strain-based and model-based modulus elastography, Ultrasound Med. Biol., 31 (2005), pp. 787-802.

[12] M. S. Gockenbach And A. A. Khan, Identification of Lamé parameters in linear elasticity: A fixed point approach, J. Indust. Manag. Optim., 1 (2005), pp. 487-497.

[13] M. Green, L. Bilston, And R. Sinkus, In vivo brain viscoelastic properties measured by magnetic resonance elastography, NMR Biomed., 21 (2008), pp. 755-764.

[14] U. Hamhaber, I. Sack, S. Papazoglou, J. Rump, D. Klatt, and J. Braun, Threedimensional analysis of shear wave propagation observed by in vivo magnetic resonance elastography of the brain, Acta Biomater., 31 (2007), pp. 127-137.

[15] L. Huwart, C. Sempoux, E. Vicaut, N. Salameh, L. Annet, E. Danse, F. Peeters, L. ter Beek, J. Rahier, R. Sinkus, Y. Horsmans, and B. Van Beers, Magnetic resonance elastography for the noninvasive staging of liver fibrosis, Gastroenterol., 135 (2008), pp. 3240.

[16] D. S. Jones, A uniqueness theorem in elastodynamics, Quart. J. Mech. Appl. Math., 37 (1984), pp. 121-142.

[17] R. KRess, Linear Integral Equations, Springer-Verlag, Berlin, 1989.

[18] S. Kruse, G. Rose, K. Glaser, A. Manduca, J. Felmlee, C. Jack, Jr., and R. Ehman, Magnetic resonance elastography of the brain, NeuroImage, 39 (2008), pp. 231-237.

[19] V. D. Kupradze, T. G. Gegelia, M. O. Basheleishvili, and T. V. Burchuladze, ThreeDimensional Problems of the Mathematical Theory of Elasticity and Thermoelasticity, North-Holland Ser. Appl. Math. Mech. 25, North-Holland, Amsterdam, 1979.

Copyright (C) by SIAM. Unauthorized reproduction of this article is prohibited. 
[20] B. Larrat, Q. Chan, X. Yang, G. Li, E. Yang, M. Fink, and R. Sinkus, Anisotropic viscoelastic properties of the corpus callosum-Application of high-resolution $3 d \mathrm{MR}$ elastography to an Alzheimer mouse model, in Proceedings of the IEEE Ultrasonics Symposium, New York, 2007, IEEE Press, Piscataway, NJ, pp. 676-679.

[21] J. LEE AND A. K. MAL, A volume integral equation technique for multiple scattering problems in elastodynamics, Appl. Math. Comput., 67 (1995), pp. 135-159.

[22] J. Lorenzen, R. Sinkus, M. Lorenzen, M. Dargatz, C. Leussler, P. Róschmann, and G. AdAm, MR elastography of the breast: Preliminary clinical results, Rofo, 174 (2002), pp. 830-834.

[23] A. Manduca, T. Oliphant, M. Dresner, J. Mahowald, S. Kruse, E. Amromin, J. Felmlee, J. Greenleaf, and R. Ehman, Magnetic resonance elastography: Non-invasive mapping of tissue elasticity, Med. Image Anal., 5 (2001), pp. 237-254.

[24] Y. K. Mariappan, K. J. Glaser, and R. L. Ehman, Magnetic resonance elastography: A review, Clinical Anatomy, 23 (2010), pp. 497-511.

[25] J. McLaughlin and D. Renzi, Shear wave speed recovery in transient elastography and supersonic imaging using propagating fronts, Inverse Problems, 22 (2006), pp. 681-706.

[26] J. R. McLaughlin, D. Renzi, and J.-R. Yoon, Anisotropy reconstruction from wave fronts in transversely isotropic acoustic media, SIAM J. Appl. Math., 68 (2007), pp. 24-42.

[27] S. G. Mikhlin And S. Prössdorf, Singular Integral Operators, Springer-Verlag, Berlin, 1986.

[28] D. Murio, The Mollification Method and the Numerical Solution of Ill-Posed Problems, John Wiley \& Sons, New York, 1993.

[29] R. Muthupillai, D. J. Lomas, P. J. Rossman, J. F. Greenleaf, A. Manduca, and R. L. EHMAN, Magnetic resonance elastography by direct visualization of propagating acoustic strain waves, Science, 29 (1995), pp. 1854-1857.

[30] G. Nakamura, Y. Jiang, S. Nagayasu, and J. Cheng, Inversion analysis for magnetic resonance elastography, Appl. Anal., 87 (2008), pp. 165-179.

[31] G. Nakamura, K. Tanuma, and G. Uhlmann, Layer stripping for a transversely isotropic elastic medium, SIAM J. Appl. Math., 59 (1999), pp. 1879-1891.

[32] G. Nakamura and G. Uhlmann, Inverse problems at the boundary for an elastic medium, SIAM J. Math. Anal., 26 (1995), pp. 263-279.

[33] A. A. Oberai, N. H. Gokhale, M. M. Doyley, and J. C. Bamber, Evaluation of the adjoint equation-based algorithm for elasticity imaging, Inverse Problems, 49 (2004), pp. 29552974.

[34] P. Olsson, The rigid movable inclusion in elastostatics and elastodynamics, Wave Motion, 7 (1985), pp. 421-445

[35] P. Olsson And D. J. N. Wall, Partial elastodynamic cloaking by means of fiber-reinforced composites, Inverse Problems, 27 (2011), pp. 1-21.

[36] A. Samani And D. Plewes, An inverse problem solution for measuring the elastic modulus of intact ex vivo breast tissue tumours, Phys. Med. Biol., 52 (2007), pp. 1247-1260.

[37] A. Samani, J. Zubovits, And D. Plewes, Elastic moduli of normal and pathological human breast tissues: An inversion-technique-based investigation of 169 samples, Phys. Med. Biol., 52 (2007), pp. 1565-1576.

[38] P. R. ShORTEN AND D. J. N. WALl, Fluid velocity profile reconstruction for non-Newtonian shear dispersive flow, J. Appl. Math. Decis. Sci., 5 (2001), pp. 87-104.

[39] R. Sinkus, J. Lorenzen, D. Schrader, M. Lorenzen, M. Dargatz, and D. Holz, Highresolution tensor MR elastography for breast tumor detection, Phys. Med. Biol., 45 (2000), pp. 1649-1664.

[40] R. Sinkus, K. Siegmann, T. Xydeas, M. Tanter, C. Claudssen, and M. Fink, MR elastography of breast lesions: Understanding the solid/liquid duality can improve the specificity of contrast-enhanced MR mammography, Magnet. Res. Med., 58 (2007), pp. 1135-1144.

[41] T. TAKenaka, D. J. N. Wall, H. Harada, and M. Tanaka, Reconstruction algorithm of the refractive index of a cylindrical object from the intensity measurements of the total field, Microw. Opt. Technol. Lett., 14 (1997), pp. 182-188.

[42] M. Tanter, J. Bercoff, A. Athanasiout, T. Deffieux, J.-L. Gennisson, G. Montaldo, M. Muller, A. TARdivon, AND M. FinK, Quantitative assessment of breast lesion viscoelasticity: Initial clinical results using supersonic shear imaging, Ultrasound Med. Biol., 34 (2008), pp. 1373-1386.

[43] F. Tréves, Introduction to Pseudodifferential Operators and Fourier Integral Operators, Vol. I, Plenum Press, New York, 1980.

[44] G. Uhlmann, J.-N. Wang, And C.-T. Wu, Reconstruction of inclusions in an elastic body, J. Math. Pures Appl., 91 (2009), pp. 569-582. 
[45] E. E. Van Houten, M. M. Doyley, F. E. Kennedy, J. B. Weaver, and K. Paulsen, Initial in vivo experience with steady-state subzone-based MR elastography of the human breast, J. Magnet. Res. Imaging, 17 (2003), pp. 72-85.

[46] E. E. W. Van Houten, M. M. Doyley, F. E. Kennedy, K. D. Paulsen, and J. B. Weaver, A three-parameter mechanical property reconstruction method for MR-based elastic property imaging, IEEE Trans. Med. Imaging, 24 (2005), pp. 311-324.

[47] E. E. W. Van Houten, D. vR. Viviers, M. D. J. McGarry, P. R. Perriñez, I. I. Perreard, J. B. WeAver, AND K. PAulsen, Subzone based magnetic resonance elastography using a Rayleigh damped material model, Med. Phys., 38 (2011), pp. 1993-2004.

[48] D. J. N. WALL, Uniqueness theorems for the inverse problem of elastodynamic boundary scattering, IMA J. Appl. Math., 44 (1990), pp. 221-241.

[49] D. J. N. WALl AND J. LundstedT, Inverse source problems involving the one-way wave equation: Medium function reconstruction, Math. Comput. Simul., 50 (1999), pp. 489-510.

[50] J. Wuerfel, F. Paul, B. Beierbach, U. Hamhaber, D. Klatt, S. Papazoglou, F. Zipp, P. Martus, J. Braun, and I. Sack, MR-elastography reveals degradation of tissue integrity in multiple sclerosis, NeuroImage, 49 (2010), pp. 2520-2525.

[51] L. Xu, Y. Lin, J. Han, Z. Xi, H. Shen, and P. GaO, Magnetic resonance elastography of brain tumors: Preliminary results, Acta Radiol., 48 (2007), pp. 327-330.

[52] P. Zabreyko, A. Koshelev, M. Krasnosel'skit, S. Mikhlin, L. Rakovshchik, and V. Stet'senko, Integral Equations-A Reference Text, Noordhoff International Publishing, Leyden, The Netherlands, 1968.

Copyright (c) by SIAM. Unauthorized reproduction of this article is prohibited. 